\title{
WALD TESTS OF $I(1)$ AGAINST $I(d)$ ALTERNATIVES: SOME NEW PROPERTIES AND AN EXTENSION TO PROCESSES WITH TRENDING COMPONENTS
}

\author{
By Juan J. Dolado ${ }^{a}$, Jesus Gonzalo ${ }^{a}$, And Laura Mayoral ${ }^{b *}$ \\ ${ }^{a}$ Dept. of Economics, U. Carlos III de Madrid; ' Institut d 'Análisi Económica (CSIC).
}

\begin{abstract}
This paper analyses the behaviour of a Wald-type test, i.e., the (Efficient) Fractional Dickey-Fuller (EFDF) test of $I(1)$ against $I(d), d<1$, relative to LM tests. Further, it extends the implementation of the EFDF test to the presence of deterministic trending components in the DGP. Tests of these hypotheses are important in many macroeconomic applications where it is crucial to distinguish between permanent and transitory shocks because shocks die out in $I(d)$ processes with $d<1$. We show how simple is the implementation of the EFDF in these situations and argue that, under fixed alternatives, it is preferred to the LM test in Bahadur's sense. Finally, an empirical application is provided where the EFDF approach allowing for deterministic components is used to test for long-memory in the GDP p.c. of several OECD countries, an issue that has important consequences to discriminate between alternative growth theories.
\end{abstract}

JEL Clasification: C12 C22 O40

Keywords: Deterministic components, Fractional processes, Power, Unit roots.

${ }^{*}$ Corresponding E-mail:jesus.gonzalo@uc3m.es. We are grateful to an anonymous referee for very helpful comments and to Claudio Michelacci and Bart Verspagen for making their data available to us. We also thank Javier Hidalgo, Javier Hualde, Francesc Mármol, Peter Robinson, Carlos Velasco. Financial support from the Spanish Ministry of Education through grants SEJ2006-00369, SEJ2004-04101 and SEJ2007-04325 and also from the Barcelona Economics Program of CREA is acknowledged. The usual disclaimer applies. 


\section{INTRODUCTION}

Typically, tests of $I(1)$ vs. $I(0)$ processes have problems in rejecting the null that a time series $\left\{y_{t}\right\}$ is $I(1)$ when the true DGP is a fractionally integrated, $I(d)$, process, particularly if $0.5<d<1$. This issue can have serious consequences for the analysis of the medium and long- run properties of macroeconomic and financial variables. For instance, (i) shocks could be identified as permanent when in fact they die out eventually, and (ii) two series could be considered as spuriously cointegrated when they are independent at all leads and lags (see Gonzalo and Lee, 1998). Further, these mistakes are more likely to occur in the presence of deterministic components as, e.g. in the case of trending economic variables.

In view of this problem, the goal of this paper is threefold. First, we discuss the power behavior of a recently proposed Wald test of $I(1)$ vs. $I(d), d \in[0,1)$ relative to the one achieved by well-known LM tests. In particular, we use the concept of Bahadur's asymptotic relative efficiency (henceforth, ARE; see Gourieroux and Monfort, 1995) to derive new analytical results regarding the non-centrality parameters of both types of tests under fixed alternatives. Secondly, we extend the Wald-type testing procedure, originally derived for driftless processes, to the more realistic case where deterministic components are present. Finally, we present a feasible linear single-step regression estimation approach to deal with serially correlated errors.

Specifically, we focus on a modification of the Fractional Dickey-Fuller (FDF) test by Dolado, Gonzalo and Mayoral (2002; DGM hereafter) recently proposed by Lobato and Velasco (2007; LV hereafter) which achieves a slight improvement in efficiency over the former. This test, henceforth denoted as the EFDF (efficient FDF) test, generalizes the traditional DF test of $I(1)$ against $I(0)$ processes without deterministic components to the broader framework of testing $I(1)$ against $I(d)$ with $0 \leq d<1$. The EFDF (and the FDF) test belongs to the family of Wald tests and relies upon the principle underlying the popular Dickey-Fuller (DF) approach. The idea is to test for the statistical significance of the slope coefficient, $\varphi$, by means of its t-ratio, $t_{\varphi}$, in a regression where the dependent variable and the regressor are filtered so as to become $I(0)$ under the null and the alternative hypothesis, 
respectively. ${ }^{1}$ Both DGM and LV set $\Delta y_{t}$ as the dependent variable, where $\Delta=(1-L) .^{2}$ As regards the regressor, whereas DGM choose $\Delta^{d} y_{t-1}$, LV show that $z_{t-1}(d)=(1-$ $d)^{-1}\left(\Delta^{d-1}-1\right) \Delta y_{t}$ improves the efficiency of the test. Non-rejection of $H_{0}: \varphi=0$ against $H_{1}: \varphi<0$, implies that the process is $I(1)$ and, conversely, rejection of the null implies that the process is $I(d)$.

In order to compute either $\Delta^{d} y_{t-1}$ or $z_{t-1}(d)$, an input value for $d$ is required. One could either consider a (known) simple alternative, $H_{A}: d=d_{A}<1$ or, more realistically, a composite one, $H_{1}: d<1$. We focus here on the last case where DGM and LV show that it suffices to use a $T^{\kappa}$-consistent estimate (with $\kappa>0$ ) of the true integration order to get a $N(0,1)$ limiting distribution of the resulting test-statistic.

Under a sequence of local alternatives approaching $H_{0}: d=1$ from below at a rate of $T^{-1 / 2}, \mathrm{LV}$ (2007, Theorem 1) prove that, with Gaussianity, the EFDF test is asymptotically equivalent to the uniformly most powerful invariant (UMPI) test, i.e., the LM test introduced by Robinson $(1991,1994)$ and later adapted by Tanaka (1999) for the time domain. We first show that, when the alternative is fixed, the former has a larger non-centrality parameter than the latter, in line with the standard result about the better power properties of Wald tests relative to LM tests (see Engle, 1984). Moreover, when compared to other tests of $I(1)$ vs. $I(d)$ which rely on direct inference about semiparametric estimators of $d$, the EFDF test exhibits better power properties in general, under a correct specification of the stationary short-run dynamics of the error term in the auxiliary regression. This is due to the fact that the semiparametric estimation procedures often imply larger confidence intervals of the memory parameter, in exchange for less restrictive assumptions on the error term and robustness in case of misspecification. ${ }^{3}$ By contrast, the combination of a wide

\footnotetext{
${ }^{1}$ In the DF setup, these filters are $\Delta=(1-L)$ and $\Delta^{0} L=L$, so that the regressand and regressor are $\Delta y_{t}$ and $y_{t-1}$, respectively.

${ }^{2}$ As shown in DGM (2002), both regressors can be constructed by applying the truncated version of the binomial expansion of the filter $(1-L)^{d}$ in the lag operator $L$ to $y_{t}(t=0,1, \ldots)$, so that $\Delta_{+}^{d} y_{t}=\sum_{i=0}^{t-1}$ $\pi_{i}(d) y_{t-i}$, where $\pi_{i}(d)$ is the i-th coefficient in that expansion, defined at the end of this Introduction. In the sequel, we will refer to this truncated filter simply as $\Delta^{d}$.

${ }^{3}$ See, e.g., Velasco (1999), Robinson (2003), Abadir et al. (2005), Shimotsu and Phillips (2005) and
} 
range of semiparametric estimators for the input value of $d$ with an auxiliary parametric regression, as the one discussed above, yields a parametric rate for the Wald tests. Thus, in a sense, the Wald tests combine the favorable features of both approaches to improve power, while reducing the danger of misspecifying short-run dynamics.

Next, we investigate how to implement the EFDF test when some deterministic components are considered in the DGP, a case which was neither considered by LV nor by DGM. Although we will analyze other types of trends, we will mainly focus on the role of a linear trend since many (macro) economic time series exhibit this type of trending behavior in their levels. Our main result is that, in contrast with what happens with most tests for $I(1)$ against $I(0)$, the EFDF test remains being efficient in the presence of deterministic components and it maintains the same asymptotic distribution, insofar as they are correctly filtered. In this respect, this result mimics the one found for LM tests in this case; cf. Robinson (1994), Tanaka (1999) and Gil-Alaña and Robinson (1997).

Lastly, we extend the results obtained for a DGP with i.i.d. error terms to the case where they are autocorrelated, as in the (augmented) DF case (ADF henceforth). In this respect, DGM (2002, Theorems 6 and 7) have proved that, in order to remove the autocorrelation, it is sufficient to augment the set of regressors in the auxiliary regression of the FDF test with $k$ lags of the dependent variable such that $k \uparrow \infty$ as $T \uparrow \infty$, and $k^{3} / T \uparrow 0$, as in Said and Dickey (1984). This leads to the augmented FDF (AFDF) test. As regards the EFDF test, we conjecture that a similar result holds, although we will confine our discussion below, as in LV (2007), to the case of finite-lag AR processes. The procedure based on the EFDF test turns out to be much simpler than accounting for serial correlation in the LM framework. Further, we point out that the two-step procedure proposed by LV (2007) can be simplified to a feasible linear single-step estimation approach. An empirical application dealing with testing the possibility that long GNP per capita series for several OECD countries may follow nonstationary $I(d)$ processes, yet with shocks that die out (supporting the hypothesis of beta-convergence) instead of $I(1)$ (no convergence), illustrates our proposed methodology.

The rest of the paper is structured as follows. Sections 2 briefly overviews the properties Shimotsu (2006). 
of the EFDF test when the process is a driftless random walk under the null and derives new results about the power of this test relative to the LM test under fixed alternatives using Bahadur's ARE. Section 3 extends the previous results to the case where the process contains trending deterministic components (e.g., a linear trend), considering both the case of i.i.d. and autocorrelated errors. Section 4 discusses an empirical application of the previous test. Finally, Section 5 draws some concluding remarks.

Proofs of the theorems are collected in the Appendix.

In the sequel, the definition of a $I(d)$ process that we will adopt is the one used by Akonom and Gourieroux (1987) where a fractional process is initialized at the origin. This corresponds to Type-II fractional Brownian motion (see the previous discussion in footnote 3 ) and is similar to the definitions of an $I(d)$ process underlying the LM test proposed Robinson (1994) and Tanaka (1999). Moreover, the following conventional notation is adopted throughout the paper: $\Gamma($.$) denotes the Gamma function, and \left\{\pi_{i}(d)\right\}$ represents the sequence of coefficients associated to the expansion of $(1-L)^{d}$ in powers of $L$,

$$
\pi_{i}(d)=\frac{\Gamma(i-d)}{\Gamma(-d) \Gamma(i+1)}
$$

The indicator function is denoted by $1_{(.)}$. Finally, $\stackrel{w}{\rightarrow}$ denotes weak convergence in $D[0,1]$ endowed with the Skorohod $J_{1}$ topology, and $\stackrel{p}{\rightarrow}$ means convergence in probability.

\section{THE EFDF TEST}

\subsection{Definitions}

Like Robinson (1994) we consider a process $\left\{y_{t}\right\}$ that is generated by an additive model, namely as the sum of a deterministic component, $\mu(t)$, and an $I(d)$ component, $u_{t}$, so that

$$
y_{t}=\mu(t)+u_{t}
$$

where $u_{t}=\Delta^{-d} \varepsilon_{t} 1_{t>0}$ is a purely stochastic $I(d)$ process and $\varepsilon_{t}$ is a zero-mean i.i.d. random variable. 
When $\mu(t) \equiv 0,{ }^{4}$ DGM introduced a Wald-type (FDF) test for testing the null hypothesis of $H_{0}: d=1$ against the composite alternative $H_{1}: 0 \leq d<1$, based on the t-ratio associated to the hypothesis $\phi=0$ in the OLS regression

$$
\Delta y_{t}=\phi \Delta^{d^{*}} y_{t-1}+v_{t}
$$

where $d^{*} \geq 0$ is an input value needed to perform the test. If $d^{*}$ is chosen such that $d^{*}=\widehat{d}_{T}$, where $\widehat{d}_{T}$ is a $T^{\kappa}$-consistent estimator of $d$, with $\kappa>0$, DGM (2002) and LV (2006) have shown that the asymptotic distribution of the resulting t-statistic, $t_{\phi}$ is $N(0,1)$.

Recently, LV (2007) have proposed the EFDF test based on a modification of regression (2) that permits to achieve higher efficiency s under the assumption of $\mu(t) \equiv 0$ (or known). More specifically, their proposal is to compute the t-statistic, $t_{\varphi}$, associated to the null hypothesis $\varphi=0$ in the regression

$$
\Delta y_{t}=\varphi z_{t-1}\left(d^{*}\right)+\varepsilon_{t}
$$

where $z_{t-1}\left(d^{*}\right)$ is defined as ${ }^{5}$

$$
z_{t-1}\left(d^{*}\right)=\frac{\left(\Delta^{d^{*}-1}-1\right)}{\left(1-d^{*}\right)} \Delta y_{t},
$$

such that $\varphi=\left(d^{*}-1\right)$. Note that, when $\varphi=0$, the model becomes a random walk, i.e., $\Delta y_{t}=\varepsilon_{t}$, while, when $\varphi=\left(d^{*}-1\right)<0$, it becomes a pure fractional process, $\Delta^{d} y_{t}=\varepsilon_{t}$. The insight for the higher efficiency of the EFDF test is that, under $H_{1}$, the regression model considered in (2) can be written as $\Delta y_{t}=\Delta^{1-d} \varepsilon_{t}=\varepsilon_{t}+(d-1) \varepsilon_{t-1}+0.5 d(d-$ 1) $\varepsilon_{t-2}+\ldots=\phi \Delta^{d} y_{t-1}+\varepsilon_{t}+0.5 d(d-1) \varepsilon_{t-2}+\ldots$ with $\phi=d-1$. Thus, the error term $v_{t}$ $=\varepsilon_{t}+0.5 d(d-1) \varepsilon_{t-2}+\ldots$ in $(2)$ is serially correlated. Although OLS provides a consistent estimator of $\phi$, since $v_{t}$ is orthogonal to the regressor $\Delta y_{t-1}=\varepsilon_{t-1}$, it is not the most efficient one. By contrast, the regression model used in the EFDF test does not suffer from this problem since, by construction, yields an i.i.d. error term. Finally, note that

\footnotetext{
${ }^{4}$ Alternatively, $\mu(t)$ could be considered to be known. In this case, the same arguments go through after substracting it from $y_{t}$ to obtain a purely stochastic process.

${ }^{5}$ A similar model was first proposed by Granger (1986) in the more general context of testing for cointegration with multivariate series, a modification of which has been recently considered by Johansen (2005).
} 
application of $\mathrm{L}^{\prime}$ Hôpital rule to $z_{t-1}\left(d^{*}\right)$ in the limit case as $d^{*} \rightarrow 1$ leads to a regressor equal to $-\ln (1-L) \Delta y_{t}=\Sigma_{j=1}^{\infty} j^{-1} \Delta y_{t-j}$, which is the one used in Robinson's LM test (see section 2.3).

Theorem 1 in LV (2007), which we reproduce below for completeness, establishes the asymptotic properties of $t_{\varphi}$.

Theorem 1 Under the assumption that the DGP is given by $y_{t}=\Delta^{-d} \epsilon_{t} 1_{(t>0)}$, $\varepsilon_{t}$ is i.i.d. with finite fourth moment, the asymptotic properties of the $t$-statistic $t_{\varphi}$ for testing $\varphi=0$ in (3), where the input of $z_{t-1}\left(\widehat{d_{T}}\right)$ is a $T^{\kappa}$-consistent estimator of $d^{*}$, for some $d^{*}>0.5$ with $\kappa>0$, are given by

a) Under the null hypothesis $(d=1)$,

$$
t_{\varphi}\left(\widehat{d}_{T}\right) \stackrel{w}{\rightarrow} N(0,1)
$$

b) Under local alternatives, $(d=1-\gamma / \sqrt{T})$,

$$
t_{\varphi}\left(\widehat{d}_{T}\right) \stackrel{w}{\rightarrow} N\left(-\gamma h\left(d^{*}\right), 1\right)
$$

where $h\left(d^{*}\right)=\Sigma_{j=1}^{\infty} j^{-1} \pi_{j}\left(d^{*}-1\right) / \sqrt{\sum_{j=1}^{\infty} \pi_{j}\left(d^{*}-1\right)^{2}}, d^{*}>0.5, d^{*} \neq 1$.

c) Under fixed alternatives $(d \in[0,1)<1)$, the test based on $t_{\varphi}\left(\widehat{d}_{T}\right)$ is consistent.

LV (2007) show that the function $h($.$) achieves a global maximum at 1$ where $h(1)=$ $\sqrt{\pi^{2} / 6}$, and that $h(1)$ equals the noncentrality parameter of the locally optimal Robinson's LM test (see subsection 2.2 below). Thus, insofar as a $T^{\kappa}$-consistent estimator of $d$ is used as input of $z_{t-1}\left(d^{*}\right)$ with $\kappa>0$, the EFDF test is locally asymptotically equivalent to Robinson's LM test. In practice, the estimate of $d$ could be smaller than 0.5. If such is the case, the input value can be chosen according to the following rule: $\widetilde{d}_{T}=\max \left\{\widehat{d}_{T}, 0.5+\vartheta\right\}$, where $\vartheta$ is a small number, e.g., $\vartheta=0.001$. A power-rate consistent estimate of $d$ can be easily obtained by applying some semiparametric estimators. Among them, the estimators proposed by Abadir et al. (2005), Shimotsu (2006) and Velasco (1999) provide convenient choices since they also cover the case where deterministic components are present, as we do in section 3 . 


\subsection{Asymptotic relative efficiency of Wald and LM tests.}

As discussed earlier, the closer competitor to the Wald (FDF and EFDF) tests is the LM test proposed by Robinson $(1991,1994)$ in the frequency domain, subsequently extended by Tanaka (1999) to the time domain. In this section we discuss the power properties of the three competing tests under fixed alternatives. ${ }^{6}$ The comparison is done in Bahadur's ARE sense.

We start with the LM test, denoted as $L M_{T}$, which considers the null hypothesis of $\theta=0$ against the alternative $\theta \neq 0$ for the DGP $\Delta^{d_{0}+\theta} y_{t}=\varepsilon_{t}$. In line with the hypotheses considered in this paper, we will focus on the particular case where $d_{0}=1$ and $-1 \leq \theta<0$. Assuming that $\varepsilon_{t} \sim N\left(0, \sigma^{2}\right)$, the score-LM test is computed as

$$
L M_{T}=\sqrt{\frac{6}{\pi^{2}}} T^{1 / 2} \sum_{j=1}^{T-1} j^{-1} \widehat{\rho}_{j} \stackrel{w}{\rightarrow} N(0,1),
$$

where $\widehat{\rho}_{j}=\sum{ }_{t=j+1}^{T} \Delta y_{t} \Delta y_{t-j} / \sum_{t=1}^{T}\left(\Delta y_{t-j}\right)^{2}$ (see Robinson, 1991 and Tanaka, 1999). Breitung and Hassler (2002) have shown that an alternative simpler way to compute the score-LM test is as the t-ratio $\left(t_{\lambda}\right)$ of $\widehat{\lambda}_{\text {ols }}$ in the regression

$$
\Delta y_{t}=\lambda x_{t-1}^{*}+e_{t}
$$

where $x_{t-1}^{*}=\sum_{j=1}^{t-1} j^{-1} \Delta y_{t-j}$. Intuitively, since $t_{\lambda}=\sum\left(\Delta y_{t} x_{t-1}^{*}\right) / \widehat{\sigma}_{e}\left(\sum\left(x_{t-1}^{*}\right)^{2}\right)^{1 / 2}$ and, under $H_{0}: \theta=0$, $\widehat{\sigma}_{e}$ tends to $\sigma$ and $\operatorname{plim} T^{-1} \sum\left(x_{t-1}^{*}\right)^{2}=\pi^{2} / 6$, then $t_{\lambda}$ has the same limiting distribution as $L M_{T}$.

Under a sequence of local alternatives of the type $\theta=\gamma T^{-1 / 2}$ with $\gamma>0$ for $H_{0}$ : $d_{0}=1$, the $L M_{T}\left(\right.$ or $\left.t_{\lambda}\right)$ is the UMPI test. However, as discussed above, the EFDF test is asymptotically equivalent to the UMPI under the appropriate choice of $\widehat{d}_{T}$ earlier discussed. Hence, as stated in Theorem 1 above, when $\mu(t) \equiv 0$ (or known) and $d=1-\gamma T^{-1 / 2}$, the limiting distribution of the the EFDF test is identical to that of the LM test, i.e., $N(-\gamma h(d), 1)$ where $h($.$) is \pi / \sqrt{6}$ for $d=1$. DGM $(2002$, Theorem 3$)$ in turn obtained that

\footnotetext{
${ }^{6}$ The available results in the literature only establish the consistency of the Wald and LM test under fixed alternatives. Yet, they do not derive the non-centrality parameters as we do below.
} 
the corresponding distribution of the FDF under local alternatives test is $N(-\gamma, 1)$. Hence, in the case of local alternatives, the asymptotic efficiency of the FDF test relative to the $\mathrm{LM}$ and EFDF tests is $0.78(\simeq \sqrt{6} / \pi)$.

In the rest of this section, we analyze the case with fixed alternatives where, to our knowledge, results are new. In particular, we derive the non-centrality parameters of the FDF, EFDF and LM tests under an $I(d)$ alternative where the DGP is assumed to be $\Delta^{d} y_{t}=\varepsilon_{t}$ with $d \in(0,1)$. Hence, $\Delta y_{t}=\Delta^{-b} \varepsilon_{t}$ where $b=d-1<0$. These non-centrality parameters correspond (in square terms) to the approximate slopes of the tests in Bahadur's sense. The following result holds.

Theorem 2 If $\Delta^{d} y_{t}=\varepsilon_{t}$ with $d \in[0,1)$, the t-statistics associated to the EFDF and FDF tests, denoted as $t_{\varphi}$ and $t_{\phi}$, respectively, verify,

$$
\begin{gathered}
T^{-1 / 2} t_{\varphi} \stackrel{p}{\rightarrow}-\left(\frac{\Gamma(3-2 d)}{\Gamma^{2}(2-d)}-1\right)^{1 / 2} \equiv c_{E F D F}(d), \\
T^{-1 / 2} t_{\phi} \rightarrow-\frac{p}{\left[\Gamma(3-2 d)-(d-1)^{2} \Gamma^{2}(2-d)\right]^{1 / 2}} \equiv c_{F D F}(d),
\end{gathered}
$$

while, under the same DGP, the LM test defined in (4) satisfies that,

$$
T^{-1 / 2} L M_{T} \stackrel{p}{\rightarrow}-\sqrt{\frac{6}{\pi^{2}}} \frac{\Gamma(2-d)}{(1-d) \Gamma(d-2)} \sum_{j=1}^{\infty} \frac{\Gamma(j+d-1)}{j \Gamma(j+2-d)} \equiv c_{L M}(d),
$$

where $c_{E F D F}(d), c_{F D F}(d)$ and $c_{L M}(d)$ denote the non-centrality parameter under the fixed alternative $d \in(0,1)$ of the EFDF, FDF and LM tests, respectively.

Figure 1 displays the three non-centrality parameters for $d \in(0,1)$. In Bahadur's sense, the ratio of the approximate slopes of the tests (e.g., $A R E(E F D F, L M, d)=\left[c_{E F D F}(d) /\left(c_{L M}(d)\right]^{2}\right)$ defines the asymptotic relative efficiency $(A R E)$ of one test versus the other. When ARE is greater than 1, it is said that the first test is asymptotically preferred (or asymptotically more powerful) in Bahadur's sense to (than) the second one (see section 23.2.3 in Gourieroux and Monfort, 1995). As expected, the noncentrality parameters of the EFDF and the LM tests behave similarly for values of $d$ very close to $H_{0}$, whereas the one of the FDF test is slightly smaller for these local alternatives. Nonetheless, the LM test performs 
significantly worse than both Wald-type tests when the alternative is not local. The EFDF tests performs slightly better than the FDF test in line with LV's (2007) arguments about efficiency. The intuition for the worse power performance of the LM test is that there does not exist any value for $\lambda$ in $(5)$ that makes $e_{t}$ both i.i.d. and independent of the regressor for fixed alternatives, implying that $x_{t-1}^{*}$ does not maximize the correlation with $\Delta y_{t}$. Figure 2 depicts the Bahadur's ARE of the EFDF and FDF tests with respect to the LM, plus the one between the two Wald tests, in the range $d \in(0.5,1)$. The message to be drawn from this Figure is similar to that in Figure 1.

In sum, for fixed alternatives with (approximately) $d<0.9$, using the above ARE criteria, these tests can be ranked in decreasing asymptotic power order as EFDF $>$ FDF $>$ LM.

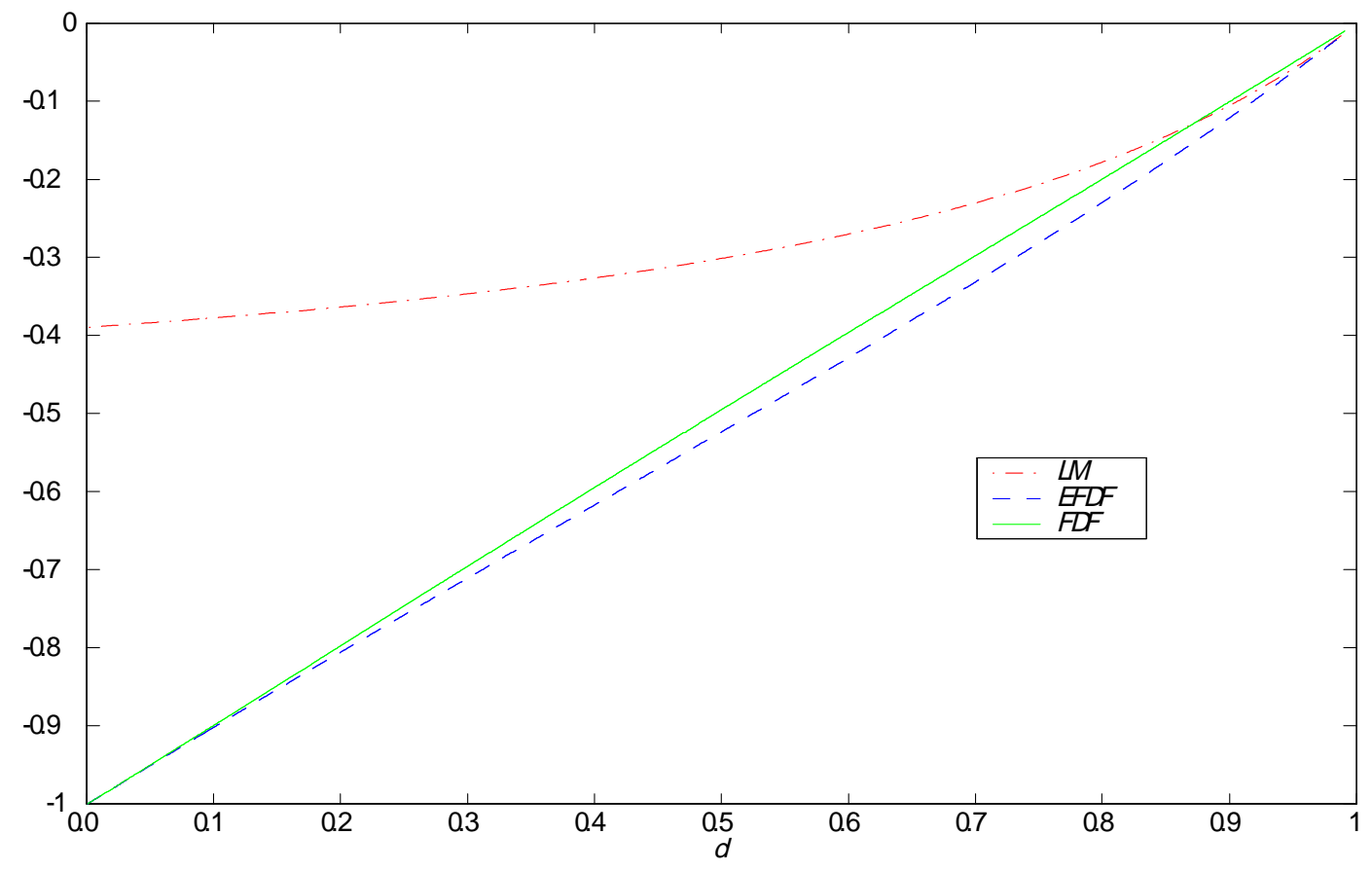

FIG 1. Non-centrality parameters of LM and Wald tests 


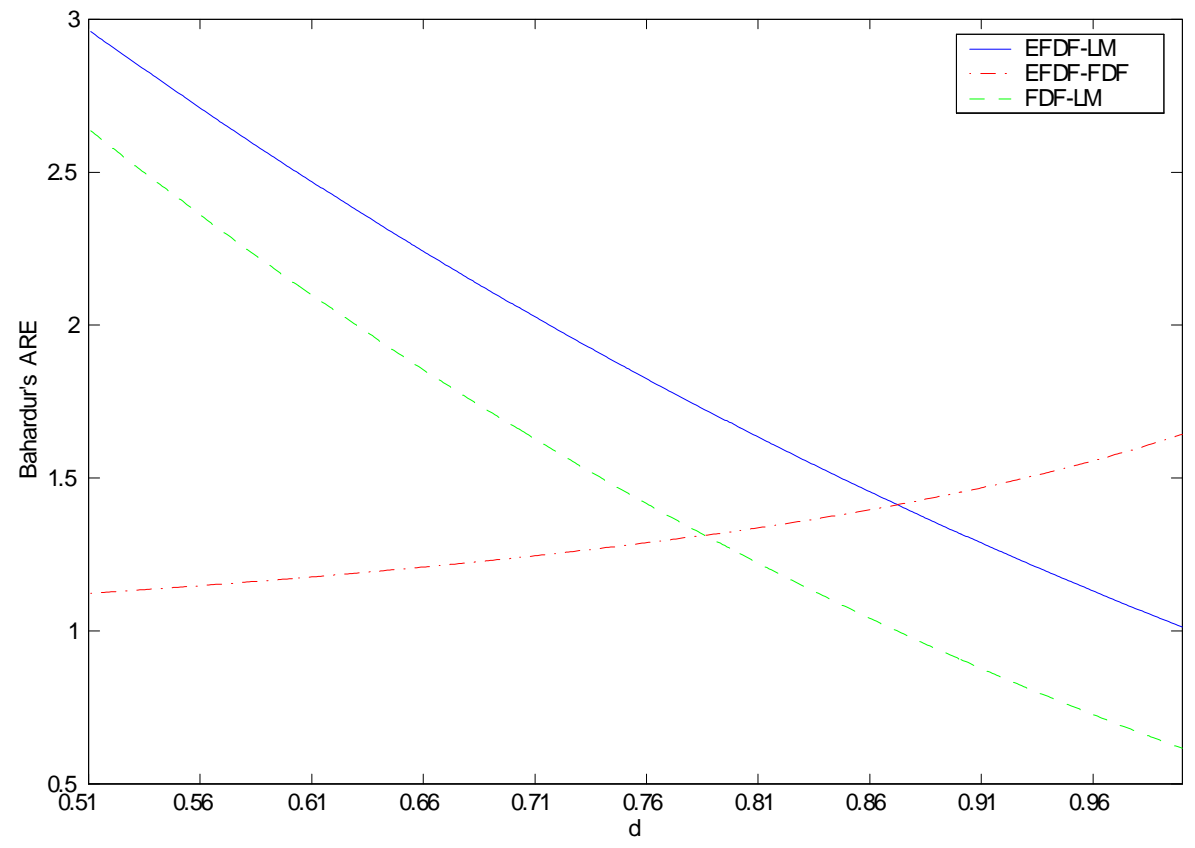

FIG 2. Bahadur's Asymptotic Relative Efficiency

As regards semiparametric estimators, both the Fully Extended Local Whittle (FELW, see Abadir et al., 2005) and the Exact Local Whittle estimators (ELW, see Shimotsu, 2006) verify the asymptotic property $\sqrt{m}\left(\widehat{d}_{T}-d\right) \stackrel{w}{\rightarrow} N\left(0, \frac{1}{4}\right)$ for $m=o\left(T^{\frac{4}{5}}\right)$. Test statistics for unit roots are based on $\tau_{d}=2 \sqrt{m}\left(\widehat{d}_{T}-1\right) \stackrel{w}{\rightarrow} N(0,1)$. Therefore, their rate of divergence under $H_{1}: d<1$ is the nonparametric rate $O_{p}(\sqrt{m})$ which is smaller than the $O_{p}(\sqrt{T})$ parametric rate achieved by the Wald test. Of course, this loss of power is just the counterpart of their higher robustness against misspecification.

\section{THE EFDF TEST FOR TRENDING I(D) PROCESSES}

\section{1 i.i.d. case}

In this section, we extend the EFDF testing approach to the more realistic case where $\mu(t) \neq 0$ and unknown. Our goal is to examine how this (unknown) deterministic term 
should be taken into account when implementing the test.

Following Elliott et al. (1996), we consider two different types of $\mu(t)$.

\section{Slowly Evolving Deterministic component}

Condition A. (Slowly evolving trend). The deterministic component $\mu(t)$ verifies

$$
\mu(t)=O\left(t^{\delta}\right), \delta<0.5
$$

Condition $\mathrm{A}$ is immediately satisfied if $\mu(t)$ is a constant term but also holds for a variety of time functions, such as slowly increasing trends, (e.g., $t^{\delta}, \delta<0.5$ or $\log t$ ).

In this case, it is straightforward to show that the stochastic component in $y_{t}$ dominates the deterministic term when $T$ is large. Hence, $\mu(t)$ has no effect either on the asymptotic distribution of the t-ratio statistic or on the efficiency properties of the test in the absence of $\mu(t)$. Therefore, one can proceed to run regression (3) ignoring the presence of these slowly evolving trends.

The following theorem presents the properties of the EFDF test when the DGP is given by $(1)$ and $\mu(t)$ verifies Condition $\mathrm{A}$.

Theorem 3 (Slowly evolving trends) Under the assumption that the DGP is given by $y_{t}=$

$\mu(t)+\Delta^{-d} \epsilon_{t} 1_{(t>0)}$, where $d \leq 1, \epsilon_{t}$ is i.i.d. with finite fourth moment, and $\mu(t)$ verifies Condition A, the asymptotic properties of the t-statistic $t_{\varphi}$ for testing $\varphi=0$ in (3) (denoted by EFDF $F_{\mu}$ test $)$, where the input of $z_{t-1}\left(\widehat{d}_{T}\right)$ is a $T^{\kappa}$-consistent estimator of $d^{*}$, for some $d^{*}>0.5$ with $\kappa>0$, are identical to those stated in Theorem 1.

\section{Evolving Deterministic Components}

Condition B. (Evolving trend). The deterministic component $\mu(t)$ verifies.

$$
\mu(t)=O\left(t^{\delta}\right), \delta \geq 0.5
$$

with $\delta$ known.

Under Condition B, the DGP is allowed to contain trending regressors in the form of, say polynomials (of known order) of $t$. Hence, when the coefficients of $\mu(t)$ are unknown, 
the test described above are unfeasible. Nevertheless, it is still possible to obtain a feasible test with the same asymptotic properties as in Theorem 1 if a consistent estimate of $\mu(t)$ is removed from the original process. Indeed, under $H_{0}$, the relevant coefficients of $\mu(t)$ can be consistently estimated by OLS in a regression of $\Delta y_{t}$ on $\Delta \mu(t)$. For instance, consider the case where the DGP contains a linear time trend, that is,

$$
y_{t}=\alpha+\beta t+\Delta^{-d} \epsilon_{t},
$$

which, under $H_{0}: d=1$, corresponds to the popular random walk with drift case. Taking first differences, it follows that $\Delta y_{t}=\beta+\Delta^{1-d} \varepsilon_{t}$. The OLS estimate of $\beta, \hat{\beta}$, (i.e., the sample mean of $\left.\Delta y_{t}\right)$ is consistent under both $H_{0}$ and $H_{1}$. In effect, under $H_{0}, \hat{\beta}$ is a $T^{1 / 2}$ -consistent estimator of $\beta$ whereas, under $H_{1}$, it is $T^{3 / 2-d}$-consistent with $3 / 2-d>0.5$ (see Hosking 1996, Theorem 8). Hence, the following theory holds.

Theorem 4 (Evolving trends) Under the assumption that the DGP is given by $y_{t}=\mu(t)+$ $\Delta^{-d} \epsilon_{t} 1_{(t>0)}$, where $d \leq 1, \epsilon_{t}$ is i.i.d. with finite fourth moment, and $\mu(t)$ satisfies Condition $B$, the asymptotic properties of the $t$ - statistic $t_{\varphi}$ for testing $\varphi=0$ in the regression

$$
\widetilde{\Delta y_{t}}=\widetilde{\varphi z_{t-1}}\left(\hat{d}_{T}\right)+e_{t}
$$

(denoted by EFDF $F_{\tau}$ test), where the input $\hat{d}_{T}$ of $\widetilde{z_{t-1}}\left(\hat{d}_{T}\right)$ is a $T^{\kappa}-$ consistent estimator of $d^{*}>0.5$ with $\kappa>0, \widetilde{\Delta y_{t}}=\Delta y_{t}-\Delta \hat{\mu}(t), \widetilde{z_{t-1}}\left(\hat{d}_{T}\right)=\frac{\left(\Delta^{\hat{d}_{T}-1}-1\right)}{\left(1-\hat{d}_{T}\right)}\left(\Delta y_{t}-\Delta \hat{\mu}(t)\right)$, and the coefficients of $\Delta \hat{\mu}(t)$ are estimated by an OLS regression of $\Delta y_{t}$ on $\Delta \mu(t)$, then the asymptotic properties of the t-statistic $t_{\varphi}$ for testing $\varphi=0$ in (7) are identical to those stated in Theorem 1.

As mentioned above, Shimotsu's (2006) semiparametric estimator provides power rate consistent estimators of $d \leq 1$ for the case where the DGP contains a linear or a quadratic trend whereas Velasco's (1999) estimator is invariant to a linear (and possibly higher order) time trend. 


\subsection{Serial correlation case: The invariant AEFDF test}

Next, we generalize the DGP considered in (1) by assuming that $u_{t}$ follows an stationary linear $A R(p)$ process, namely, $\Phi_{p}(L) u_{t}=\epsilon_{t} 1_{t>0}$ where $\Phi_{p}(L)=1-\phi_{1} L-\ldots-\phi_{p} L^{p}$ with $\Phi_{p}(z) \neq 0$ for $|z| \leq 1$. This motivates the following nonlinear regression model

$$
\Delta y_{t}=\varphi\left[\Phi_{p}(L) z_{t-1}(d)\right]+\sum_{j=1}^{p} \phi_{j} \Delta y_{t-j}+\epsilon_{t},
$$

which is similar to (3), except for the inclusion of the lags of $\Delta y_{t}$ and for the filter $\Phi_{p}(L)$ in the regressor whose significance is tested. Estimation of this model is complicated because of the nonlinearity in the parameters $\varphi$ and $\Phi_{p}=\left(\phi_{1}, \ldots, \phi_{p}\right)$. Compared with the i.i.d case, the practical problem arises because the vector $\Phi$ is unknown and therefore the regressor $\left[\Phi_{p}(L) z_{t-1}(d)\right]$ is unfeasible. For this reason LV (2007) recommended to apply a two-step procedure that allows one to obtain efficient tests also with autocorrelated errors.

\subsubsection{Two-step procedure.-}

For the case where $\mu(t) \equiv 0$ (or known), LV (2007) implement the two step procedure as follows. In the first step, the coefficients of $\Phi_{p}(L)$ are estimated (under $H_{1}$ ) by OLS in the equation

$$
\Delta^{\widehat{d}_{T}} y_{t}=\sum_{t=1}^{p} \phi_{j} \Delta^{\widehat{d}_{T}} y_{t-j}+a_{t},
$$

where $\widehat{d}_{T}$ satisfies the conditions stated in Theorem 1. The estimator of $\Phi_{p}(L)$ is consistent with a convergence rate which depends on the rate $\kappa$. Second, estimate by OLS the equation

$$
\Delta y_{t}=\varphi\left[\widehat{\Phi}_{p}(L) z_{t-1}\left(\hat{d}_{T}\right)\right]+\sum_{j=1}^{p} \phi_{j} \Delta y_{t-j}+v_{t},
$$

where $\widehat{\Phi}_{p}(L)$ is the estimator from the first step, and $\widehat{d}_{T}$ denotes the same estimated input used in that step as well. As LV (2007, Theorem 2) have shown, the $t_{\varphi}$ statistic in this augmented regression is still both normally distributed and locally optimal. The test will be denoted by AEFDF (augmented EFDF) test in the sequel. 
For the case where the coefficients of $\mu(t)$ are considered to be unknown, a similar procedure as that described in section 2.1 can be implemented and efficient tests will still be obtained. If $\mu(t)$ is a slowly moving trend satisfying Condition A, the test based on regression (10) can be implemented and the asymptotic properties stated in LV (2007, Theorem 2 ) still hold through. For the case where $\mu(t)$ satisfies Condition B, as discussed earlier, one needs to remove these terms from the original variables prior to computing regressions (9) and (10), where the coefficients of $\mu(t)$ can be estimated by OLS under the null. For instance, if the DGP is defined as in (6), a consistent estimator of $\beta$ is obtained from the OLS estimator of a regression of $\Delta y_{t}$ on a constant term. Clearly, this estimator has the same properties in this case as those described in Section 3.1. Then, regression (9) simply becomes

$$
\Delta^{\widehat{d}_{T}}\left(y_{t}-\hat{\beta} t\right)=\left[1-\Phi_{p}(L)\right] \Delta^{\widehat{d}_{T}}\left(y_{t}-\hat{\beta} t\right)+a_{t}
$$

whereas regression (10) would be

$$
\widetilde{\Delta y_{t}}=\varphi\left[\widehat{\Phi}_{p}(L) \widetilde{z_{t-1}}\left(\hat{d}_{T}\right)\right]+\sum_{t=1}^{p} \phi_{j} \widetilde{\Delta y_{t-j}}+v_{t},
$$

and $\widetilde{\Delta y_{t}}=\Delta y_{t}-\hat{\beta}$ and $\widetilde{z_{t-1}}\left(\hat{d}_{T}\right)=\frac{\left(\Delta^{\hat{d}_{T}-1}-1\right)}{\left(1-\hat{d}_{T}\right)}\left(\Delta y_{t}-\hat{\beta}\right)$. In the case where $\mu(t)$ in the DGP contains a quadratic term, $\Delta y_{t}$ should be regressed on a constant and a linear time trend and so forth for higher-order time trends.

LV (2007) have shown that the asymptotic properties of the two-step AEFDF test is identical to those in Theorems 1 and 2, except that, under local alternatives $(d=1-\gamma / \sqrt{T}$, with $\gamma>0)$, we have that $t_{\varphi}(d) \stackrel{w}{\rightarrow} N(-\gamma \omega, 1)$ and $t_{\psi}(d) \stackrel{w}{\rightarrow} N(\gamma \omega, 1)$ where

$$
\omega^{2}=\frac{\pi^{2}}{6}-\varkappa^{\prime} \Psi^{-1} \varkappa
$$

such that $\varkappa=\left(\varkappa_{1}, \ldots, \varkappa_{p}\right)^{\prime}$ with $\varkappa_{k}=\sum_{j=k}^{\infty} j^{-1} c_{j-k}, k=1, \ldots, p, c_{j}$ 's are the coefficients of $L^{j}$ in the expansion of $1 / \Phi(L)$, and $\Psi=\left[\Psi_{k, j}\right], \Psi_{k, j}=\sum_{t=0}^{\infty} c_{t} c_{t+|k-j|}, k, j=1, \ldots, p$, denotes the Fisher information matrix for $\Phi(L)$ under Gaussianity. Note that $\omega^{2}$ is identical to the drift of the limiting distribution of the LM test under local alternatives (see Tanaka, 1999). 


\subsubsection{Single-step procedure.-}

In this section we show that a single-step procedure can also be applied with the same properties. Our one-step method starts by defining the following decomposition for the polynomial $\Phi_{p}(L)$

$$
\Phi_{p}(L)=\Phi_{p}(1)+\frac{1}{\Delta^{d-1}-1} \Phi_{p}^{*}(L),
$$

where the polynomial $\Phi_{p}^{*}(L)$ is defined by equating (12) to the standard Beveridge-Nelson polynomial decomposition

$$
\Phi_{p}(L)=\Phi_{p}(1)+\Delta \widetilde{\Phi}_{p}(L)
$$

By doing that we obtain

$$
\frac{1}{\Delta^{d-1}-1} \Phi_{p}^{*}(L)=\Delta \widetilde{\Phi}_{p}(L)
$$

and therefore

$$
\Phi_{p}^{*}(L)=\left(\Delta^{d}-\Delta\right) \widetilde{\Phi}_{p}(L)=\Delta^{d} \widetilde{\Phi}_{p}(L)-\left[\Phi_{p}(L)-\Phi_{p}(1)\right]
$$

Substituting (12) into (8) and using (15), yields

$$
\begin{aligned}
\Delta y_{t} & =\varphi\left[\Phi_{p}(1) z_{t-1}(d)\right]+\left[\frac{\varphi}{1-d} \Phi_{p}^{*}(L)\right] \Delta y_{t}+\left[1-\Phi_{p}(L)\right] \Delta y_{t}+\varepsilon_{t} \\
& =\varphi\left[\Phi_{p}(1) z_{t-1}(d)\right]-\left[\Delta^{d} \widetilde{\Phi}_{p}(L)-\left(\Phi_{p}(L)-\Phi_{p}(1)\right)\right] \Delta y_{t}+\left[1-\Phi_{p}(L)\right] \Delta y_{t}+\varepsilon_{t}(16)
\end{aligned}
$$

Operating we obtain the final model

$$
\Delta y_{t}=\varphi z_{t-1}(d)-\frac{1}{\Phi_{p}(1)} \widetilde{\Phi}_{p}(L) \Delta^{d+1} y_{t}+\frac{1}{\Phi_{p}(1)} \varepsilon_{t} .
$$

Finally, to have only lagged variables in the right hand side of (17), we can proceed as follows 


$$
\begin{aligned}
\Delta y_{t}= & \varphi z_{t-1}(d)-\frac{1}{\Phi_{p}(1)}\left[\widetilde{\Phi}_{p}(L)-\widetilde{\Phi}_{p}(0)+\widetilde{\Phi}_{p}(0)\right]\left[\Delta^{d}-1+1\right] \Delta y_{t}+\frac{1}{\Phi_{p}(1)} \varepsilon_{t} \\
= & \varphi z_{t-1}(d)-\frac{1}{\Phi_{p}(1)}\left\{\left[\widetilde{\Phi}_{p}(L)-\widetilde{\Phi}_{p}(0)\right]\left[\Delta^{d}-1\right] \Delta y_{t}+\left[\widetilde{\Phi}_{p}(L)-\widetilde{\Phi}_{p}(0)\right] \Delta y_{t}\right. \\
& \left.+\left[\widetilde{\Phi}_{p}(0)\right]\left[\Delta^{d}-1\right] \Delta y_{t}+\left[\widetilde{\Phi}_{p}(0)\right] \Delta y_{t}\right\}+\frac{1}{\Phi_{p}(1)} \varepsilon_{t} \\
= & \varphi z_{t-1}(d)-\frac{1}{\Phi_{p}(1)}\left\{\left[\widetilde{\Phi}_{p}(L)\right]\left[\Delta^{d}-1\right] \Delta y_{t}+\left[\widetilde{\Phi}_{p}(L)-\widetilde{\Phi}_{p}(0)\right] \Delta y_{t}\right. \\
& \left.+\left[\widetilde{\Phi}_{p}(0)\right] \Delta y_{t}\right\}+\frac{1}{\Phi_{p}(1)} \varepsilon_{t} .
\end{aligned}
$$

Therefore

$$
\begin{aligned}
\frac{\Phi_{p}(1)+\widetilde{\Phi}_{p}(0)}{\Phi_{p}(1)} \Delta y_{t}= & \varphi z_{t-1}(d)-\frac{1}{\Phi_{p}(1)}\left[\widetilde{\Phi}_{p}(L)\right]\left[\Delta^{d}-1\right] \Delta y_{t} \\
& -\frac{1}{\Phi_{p}(1)}\left[\widetilde{\Phi}_{p}(L)-\widetilde{\Phi}_{p}(0)\right] \Delta y_{t}+\frac{1}{\Phi_{p}(1)} \varepsilon_{t}
\end{aligned}
$$

so that

$$
\begin{aligned}
\Delta y_{t}= & \varphi\left[\frac{\Phi_{p}(1)}{\Phi_{p}(1)+\widetilde{\Phi}_{p}(0)}\right] z_{t-1}(d)-\frac{1}{\Phi_{p}(1)+\widetilde{\Phi}_{p}(0)}\left[\widetilde{\Phi}_{p}(L)\right]\left[\Delta^{d}-1\right] \Delta y_{t} \\
& -\frac{1}{\Phi_{p}(1)+\widetilde{\Phi}_{p}(0)}\left[\widetilde{\Phi}_{p}(L)-\widetilde{\Phi}_{p}(0)\right] \Delta y_{t}+\frac{1}{\Phi_{p}(1)+\widetilde{\Phi}_{p}(0)} \varepsilon_{t} .
\end{aligned}
$$

Noticing that $\Phi_{p}(1)+\widetilde{\Phi}_{p}(0)=1^{7}$, this model can be simplified to

$$
\Delta y_{t}=\varphi\left[\Phi_{p}(1)\right] z_{t-1}(d)-\widetilde{\Phi}_{p}(L)\left[\Delta^{d}-1\right] \Delta y_{t}-\left[\widetilde{\Phi}_{p}(L)-\widetilde{\Phi}_{p}(0)\right] \Delta y_{t}+\varepsilon_{t}
$$

where notice that neither $\left[\Delta^{d}-1\right] \Delta y_{t}$ nor $\left[\widetilde{\Phi}_{p}(L)-\widetilde{\Phi}_{p}(0)\right] \Delta y_{t}$ contain contemporaneous terms in $\Delta y_{t}$. Since $\left[\Delta^{d}-1\right] \Delta y_{t}$ can be expressed as an (infinite) lag polynomial of $\Delta y_{t-1}$, in practice regression (21) could be run regressing $\Delta y_{t}$ on $z_{t-1}(d)$ and lags $\Delta y_{t}$, using a truncation rule similar to that proposed in DGM (2002, Theorem 7). Also notice that when $\Phi_{p}(1) \simeq 0$, i.e., the gain of the autoregressive process is close to unity, the AEFDF test is

\footnotetext{
${ }^{7}$ This results follows from the Beveridge-Nelson decomposition $\Phi_{p}(L)=\sum_{0}^{\infty} \phi_{j} L^{j}=\Phi_{p}(1)+\Delta \widetilde{\Phi}_{p}(L)$, with $\phi_{0}=1$, and $\widetilde{\Phi}_{p}(L)=\sum_{0}^{\infty} \widetilde{\phi}_{j} L^{j}$, where $\widetilde{\phi}_{j}=-\sum_{j+1}^{\infty} \phi_{k}$.
} 
bound to have low power since the composed coefficient $\varphi \Phi_{p}(1)$ will be close to zero even when $\varphi<0$.

The following examples illustrate show how this procedure works in two simple cases of autocorrelated errors.

Example $1(\operatorname{AR}(\mathbf{1})): \Phi_{1}(L)=\left(1-\phi_{1} L\right)$. In this case $\Phi_{1}(1)=1-\phi_{1}, \widetilde{\Phi}_{1}(L)=\phi_{1}$, and $\widetilde{\Phi}_{1}(0)=\phi_{1}$. Then, the regression model becomes

$$
\Delta y_{t}=\varphi\left(1-\phi_{1}\right) z_{t-1}(d)-\phi_{1}\left[\Delta^{d}-1\right] \Delta y_{t}+\varepsilon_{t}
$$

Example $2(\mathbf{A R}(2)): \Phi_{2}(L)=\left(1-\phi_{1} L-\phi_{2} L\right)$. In this case $\Phi_{2}(1)=1-\phi_{1}-\phi_{2}$,

$\widetilde{\Phi}_{2}(L)=\left(\phi_{1}+\phi_{2}\right)+\phi_{2} L, \widetilde{\Phi}_{2}(0)=\phi_{1}+\phi_{2}$, and $\widetilde{\Phi}_{2}(L)-\widetilde{\Phi}_{2}(0)=\phi_{2} L$. Then, the regression (21) becomes

$$
\Delta y_{t}=\varphi\left(1-\phi_{1}-\phi_{2}\right) z_{t-1}(d)-\left[\left(\phi_{1}+\phi_{2}\right)+\phi_{2} L\right]\left[\Delta^{d}-1\right] \Delta y_{t}-\phi_{2} \Delta y_{t-1}+\varepsilon_{t} .
$$

There are two messages we can send to practitioners: (i) Regress $\Delta y_{t}$ on $z_{t-1}(d)$, on contemporaneous and lags of $\left[\Delta^{d}-1\right] \Delta y_{t}$, and lags of $\Delta y_{t}$, or (ii) Regress $\Delta y_{t}$ on $z_{t-1}(d)$ and lags of $\Delta y_{t}$, using a truncation rule as the one discussed above. The first type of regression (Full-method) has the advantages that all the lag polynomials are finite and the order can be selected consistently by some information criteria. It has the disadvantage of generated regressors because of having to estimate $d$ in order to generate the second set of regressors (this problem does not occur when we test against a simple alternative $d=d_{A}$ ). On the contrary the second type of regressions (Simple-method) does not have this problem but the lags will be infinite and therefore dependent on the truncation rule. If there are unknown deterministic components in the model, then, apply the previous tests with the deviations $\widetilde{\Delta y_{t}}$ and $\widetilde{z_{t-1}}(d)$.

\subsection{Monte Carlo evidence}

In this section we study the finite sample performance of the tests analyzed in this paper. The discussion is divided in two cases, with and without i.i.d. error terms. 


\subsection{1 i.i.d. error terms.-}

Monte-Carlo evidence in favour of the EFDF and FDF tests can be found in LV (2007) and DGM (2002), respectively, for the case where $\mu(t) \equiv 0$ and i.i.d. error terms. In what follows, we keep the i.i.d. assumption and provide some additional simulations when $\mu(t)=\alpha+\beta t$. Table 1 presents the empirical rejection frequencies for local alternatives at the $5 \%$ level of the EFDF, LM and Shimotsu's ELW tests (denoted as ELW ${ }_{\mu}$ and ELW , respectively). The DGP is $y_{t}=\alpha+\beta t+\Delta^{-d} \varepsilon_{t}$, with $\varepsilon_{t} \sim$ n.i.d $(0,1), d=1-\gamma / T^{1 / 2}$ for $\gamma=\{0,0.5,1.0,2.0$ and 5.0$\}$ and $T=\{100,400\}$. The number of simulations is $N=10,000$. Shimotsu's (2006) ELW estimator has been used for the input value of $d$, $\widehat{d}_{T}$. The figures corresponding to $\mathrm{EFDF}_{\mu}, \mathrm{LM}_{\mu}$ and ELW $\mathrm{EL}_{\mu}$ are obtained by setting $\alpha=1$, $\beta=0$, whereas those for $\mathrm{EFDF}_{\tau}, \mathrm{LM}_{\tau}$ and $\mathrm{ELW}_{\tau}$ pertain to $\alpha=1, \beta=0.2$. Inspection of the results show that, for the smaller sample sizes (when $\gamma=0$ ), the LM test is slightly under-sized whereas the EFDF and ELW test are slightly over-sized, specially when we allow for a linear trend. For this reason, we compute size-adjusted power for $\gamma>0^{8}$. The most relevant finding is that, as expected, both EFDF and LM tests have similar power for the two smaller values of $\gamma$ whereas the former has larger power for $\gamma=2$ and 5 , with improvements up to 5 percentage points in some instances. In turn, the ELW test behaves somewhat similarly to the other two tests for $\gamma=0.5$ and 1.0, whilst it loses quite a lot of power for the larger values of $\gamma$.

\section{[Table 1 about here]}

In Table 2 we also report the results of simulating the same DGP as in Table 1, except $\gamma=$ 5 , but with errors following an i.i.d. (demeaned) $\chi^{2}(1)$ distribution rather than n.i.d $(0,1)$. The reported results correspond to the $\tau$-version of the tests, reaching similar conclusions to the ones discussed earlier. ${ }^{9}$

\footnotetext{
${ }^{8}$ Response surface estimates of finite sample critical values of the EFDF under the presence of deterministic components can be found in Sephton (2007).

${ }^{9}$ Similar conclusions also hold when the error tem in the DGP follow a Student's $t$ distribution with 5 d.f.
} 


\section{[Table 2 about here]}

\subsubsection{Serially correlated error terms.-}

Table 3 presents effective size and (size-adjusted) power of the AEFDF, LM and ELW tests when the errors are autocorrelated. The DGP is now $\Delta^{d} y_{t}=\alpha+\beta t+\varepsilon_{t} /(1-0.2 L)$, with $N=10,000$ for several values of $d=1-\gamma / T^{1 / 2}$, using the the same values of $\gamma$ and $T$ as before plus combinations of $\alpha=1$ and $\beta=0.2$. The AEFDF test is implemented using model (22) on the detrended variables. Although for this $\mathrm{AR}(1)$ disturbance, power is lower than in the i.i.d. case, the comparison across the three tests is similar to the one discussed above, with the AEFDF test performing better for the larger values of $\gamma$.

\section{[Table 3 about here]}

Next, in Table 4a, we perform a comparison of the two single-step procedures discussed in section 3.2.2: simple and full methods. The DGP we consider is $\Delta^{d} y_{t}=\varepsilon_{t} /\left(1-\phi_{1} L\right)$, again with $N=10,000$ for several values of $\phi_{1}$ and $d$ in the ranges $[0,0.8]$ and $[0.6,1]$, respectively, and $T=\{100,500\}$, the sample size used in $\operatorname{LV}(2007)$. The input value of $d$ is estimated with Shimotsu's (2006) nonparametric approach. For the Simple-method we use one lag when $T=100$ and two lags for $T=500$. Full-method is based on regression (22). In general, both procedures yield similar results with some exceptions. For instance, for $T=500$ with $\phi_{1} \geq 0.6$ and $d \geq 0.7$, the Simple-method exhibits much higher power. Since a high value of $\phi_{1}$ leads to low power of the AEFDF test, this seems to lead to a substantial advantage of the Simple-method over the Full-method.

\section{[Table 4a about here]}

Finally, to gauge how LV's (2007) two-step procedure fares relative to our proposed single-step procedures, Table $4 \mathrm{~b}$ presents results on size and (size-adjusted) power of the 
former approach for an identical DGP to that used in Table $4 \mathrm{a} .{ }^{10}$ Both procedures yield similar results though, interestingly, for $\phi_{1} \geq 0.6$ and $d \geq 0.7$, the Simple-method has higher power.

\section{[Table $4 \mathrm{~b}$ about here]}

\section{EMPIRICAL ILLUSTRATION}

An interesting application of the theoretical results above is to examine whether the time-series of GDP per capita of several OECD countries behave as $I(d)$ processes with $d \in(0.5,1)$. These are series which are clearly trending upwards and therefore provide nice examples of the role of deterministic terms in the use of the EFDF test. As pointed out by Michelacci and Zaffaroni (2000; henceforth, MZ), such a long-memory behavior could well explain the seemingly contradictory results obtained in the literature on growth and convergence. The puzzling result is that a unit root cannot be rejected in (the log of) those series and yet a $2 \%$ rate convergence rate to a steady-state level (approximated by a linear trend) is typically found in most empirical exercises testing the so-called unconditional beta- convergence hypothesis (see Barro and Sala i Martín, 1995 and Jones, 1995). The explanation offered by MZ to this puzzle relies upon two well-known results in the literature on long-memory processes, namely that standard unit root tests have low power against fractional values of $d$ in the nonstationary range, and that for all values of $d \in[0,1)$ the effects of shocks die out. Notice that consideration of GDP p.c as an $I(d)$ process may be very reasonable since GDP is obtained as the aggregation of value-added in a wide range of productive sectors which are likely to have different persistence properties (see Lo and Haubrich, 2001). Thus, the aggregation argument popularized by Granger (1980) applies strongly to this case.

Using Maddison's (1995) data set of annual GDP per capita series for 16 OECD countries

\footnotetext{
${ }^{10}$ The results are (almost) identical to those reported in Table III of LV(2007).
} 
during the period 1870-1994 and the log-periodogram estimator of $d$ due to Robinson (1995), MZ find that in most countries the order of fractional integration is in the interval $(0.5,1)$, theoretically compatible with the $2 \%$ rate of convergence found in the literature of betaconvergence and, therefore, validating in this way their explanation of the puzzle. Since that estimation procedure is restricted to the range of $I(d)$ processes with finite variance, namely, $|d|<1 / 2$, MZs proceed by first detrending the data and then applying the truncated filter $(1-L)^{1 / 2}$ to the residuals, discarding the first ten observations to initialize the series.

The previous results have been criticized by Silverberg and Verspagen (2001) on the grounds that the use of the Geweke and Porter-Hudak (GPH) semi-parametric estimation procedure, as modified by Robinson (1995), suffers from serious small-sample bias. Instead, they propose to use the first-difference filter, $(1-L)$, to remove the trend, and then employ both Beran's (1994) nonparametric estimator and Sowell's (1992) parametric ML estimator of ARFIMA models to tackle short-memory contamination in the estimation of $d$. By using these estimation procedures, Silverberg and Verspagen (2001) find, in stark contrast to MZ 's results, that $d$ tends to be either not significantly different from unity or significantly above unity for most countries.

To shed light on this controversy, we apply the AEFDF test developed in Section 3.2 to the logged GDP p.c. of a subset of thirteen of the main OECD countries, listed in Table 5 , where (under the null) the estimated intercept and its (Newey-West robust) standard deviation in the regression $\Delta y_{t}=\beta+u_{t}$ is reported. ${ }^{11}$ As can be inspected, the mean (average GDP p.c. growth rate) is always highly significant making it convenient to use a model which allows for a linear trend, as in (6), as the maintained hypothesis. Indeed, when the ADF and the Phillips-Perron (P-P) unit root tests (not reported) were computed using Elliott et al. (1996)'s efficient GLS detrending procedure, the $I(1)$ null hypothesis could not be rejected in most cases ${ }^{12}$. The KPPS test, which takes $I(0)$ as the null, also

\footnotetext{
${ }^{11}$ Maddison's (2004) dataset has been employed in this case, which adds 9 observations to the data considered by MZ.

${ }^{12}$ The only exceptions are Canada, Germany and the US with p-values of $0.045,0.049$ and 0.040 , respectively.
} 
yielded rejection in more than half of the cases, confirming the high persistence of the series. Thus it seems clear that the levels of the series have a linear trend and that deviations from such a trend are likely to be nonstationary. In addition, since there were clear signs of autocorrelation in $u_{t}$, an AEFDF test was applied to the series. The number of lags of the dependent variable was chosen according to the AIC with a maximum lag of length $k=5$.

\section{[Table 5 about here]}

Pre-estimation of $d$ using Shimotsu's (2006) nonparametric approach allows one to estimate a value of $d$ for each country. The estimated values of $d$ are always in the non-stationary range. Taking into account that the standard error (s.e.) of this estimator is $\sqrt{1 / 4 m}$ with $m=T^{0.65}$, with a sample size of $T=134$, it takes a value of 0.102 in all cases. Using this s.e., the value $d=1$ is included in an appropriate confidence interval of 12 out of the 13 countries, yielding similar results to those in Silverberg and Verspagen (2001). Nevertheless, using the AEFDF test with the above-mentioned estimated input value, $\widehat{d}_{T}$, the first column of Table 6 shows strong rejections of $H_{0}: \quad d=1$ in 6 out of the 13 countries. ${ }^{13}$ As discussed earlier, the intuition for this higher rejection rate is the higher power of the EFDF test relative to pure semiparametric tests which yield wider confidence intervals. Thus, our results in almost half of the countries seem to favor nonstationary $I(d)$ processes with $d<1$, in line with MZ's conclusions. As Jones (1995) first suggested, this evidence is inconsistent with endogenous growth theories for which permanent changes in certain policy variables have permanent effects on the rate of economic growth. We are aware that a definitely conclusion on this issue requires a deeper data analysis in at least two directions: (i) testing long memory versus structural breaks, and (ii) deriving a panel version of the proposed EFDF test. Both directions are being under current investigation by the authors (for the former, see Dolado et al., 2005).

[Table 6 about here]

\footnotetext{
${ }^{13}$ When the estimated value of $d$ was larger than unity, a value of $\hat{d}_{T}=1$ was employed as an input to run the test.
} 


\section{CONCLUSIONS}

This paper provides new theoretical results regarding gains in power, under fixed alternatives, of applying a Wald test instead of the conventional LM test for detecting the presence of a unit root in time-series data against the alternative of $I(d), d<1$, possibly allowing for a wide variety of deterministic terms in the DGP. The Wald test is based on the EFDF testing approach (see LV, 2007). Four main findings have been obtained. First, though the EFDF test is asymptotically equivalent to the LM test under local alternatives, it has larger power in Bahadur's sense under fixed alternatives. This gain in power relative to the LM test may also hold for other Wald tests, like the FDF test (see DGM, 2002) which are less efficient than the EFDF test. Secondly, if $\mu(t)$ is slowly evolving trend (e.g, including just a constant term), then the EFDF test ignoring $\mu(t)$ can be implemented without losing any of its optimal asymptotic properties. Thirdly, if $\mu(t)$ is a polynomial in $t$ of known order but unknown coefficients, then these properties remain identical if one runs the EFDF test on the OLS residuals of the regression of $\Delta y_{t}$ on $\mu(t)$ under the null of $d=1$. And, fourthly, under the presence of serial correlation, we show that the EFDF test can be performed in a feasible linear single-step instead of the two-step procedure proposed by LV (2007). An empirical application regarding the issue of whether deviations from a trend of GDP p.c. in a variety of countries follow an $I(1)$ or a nonstationary $I(d)$ where shocks die out illustrates the usefulness and simplicity of the testing approach proposed here.

Interesting extensions under current investigation by the authors include testing fractional integration versus $I(0)$ allowing for structural breaks (see Dolado, Gonzalo and Mayoral, $2007)$, testing for cointegration between two $I(d)$ series which have a non-zero drift and where a constant term or a linear trend is included in the regression model and finally, an extension of this framework to panel data. 


\section{REFERENCES}

Abadir, K., Distaso, W. and L. Giraitis (2005), "Semiparametric estimation for trending $\mathrm{I}(\mathrm{d})$ and related processes," mimeo.

Akonom, J. and C. Gourieroux (1987), "A functional limit theorem for fractional processes," CEPREMAP, mimeo.

Baillie, R.T. (1996), "Long memory processes and fractional integration in economics and finance," Journal of Econometrics, 73, 15-131.

Barro, R. J. and X. Sala i Martín (1995), Economic Growth . McGraw-Hill, New York.

Beran, J. (1994), Statistics for Long Memory Processes, New York: Chapman \& Hall.

Breitung, J. and U. Hassler (2002), "Inference on the cointegrated rank of in fractionally integrated processes," Journal of Econometrics, 110, 167-185.

Davidson, J. (1994), Stochastic Limit Theory. New York: Oxford University Press.

Dolado, J., Gonzalo, J. and L. Mayoral (2002), "A fractional Dickey-Fuller test for unit roots," Econometrica, 70, 1963-2006.

Dolado, J., Gonzalo, J. and L. Mayoral (2007), "Structural breaks vs. long memory: What is what?" Universidad Carlos III, Madrid, mimeo.

Elliott, G., Rothenberg, T. and J. Stock (1996), "Efficient tests for an autoregressive unit root," Econometrica, 64, 813-836.

Engle, R.F. (1984) "Wald, Likelihood Ratio and Lagrange Multiplier tests in Econometrics," in Z. Griliches and R. Intrilligator (eds) Handbook of Econometrics, vol II, 75-826, Amsterdam: North Holland.

Gil-Alaña, L. A. and P. Robinson (1997), "Testing unit roots and other statistical hypothesis in macroeconomic time series," Journal of Econometrics, 80, 241-268.

Gonzalo, J. and T. Lee (1998), "Pitfalls in testing for long-run relationships," Journal of Econometrics, 86, 129-154.

Gourieroux, C. and A. Monfort (1995), Statistics and Econometric Models, Volume Two. Cambridge University Press.

Granger, C.W.J. (1980), "Long memory relationships and the aggregation of dynamic 
models," Journal of Econometrics, 14, 227-238.

Granger, C. W. J. (1986), "Developments in the study of cointegrated economic variables", Oxford Bulletin of Economics and Statistics, 48, 213-228.

Hosking, J.R.M., (1996) "Asymptotic distributions of the sample mean, autocovariances, and autocorrelations of long-memory time series", Journal of Econometrics, Vol. 73, (1), 261-284.

Johansen, S. (2005), "A representation theory for a class of vector autoregressive models for fractional processes"

Jones, C. (1995), "Time series tests of endogenous growth models," Quarterly Journal of Economics, 110, 495-525.

Liu M. (1998), "Asymptotics of nonstationary fractionally integrated series," Econometric Theory, 14, 641-662.

Lo, A.W. and J.G. Haubrich (2001), "The sources and nature of long-term dependence in the business cycle," Economic Review 37, 15-30.

Lobato, I. and C. Velasco (2006), "Optimal fractional Dickey-Fuller tests for unit roots," Econometrics Journal, 9, 492-510.

Lobato, I. and C. Velasco (2007), "Efficient Wald tests for fractional unit roots," Econometrica, 75, 575-589.

Maddison, A. (1995), Monitoring the World Economy, 1820-1992, Paris: OECD.

Michelacci, C. and P. Zaffaroni, P.(2000), "Fractional beta convergence," Journal of Monetary Economics, 45, 129-153.

Robinson, P.M. (1994), "Efficient tests of nonstationary hypotheses," Journal of the American Statistical Association, 89, 1420-1437.

Robinson, P. M. (1995), "Log-periodogram of time series with long-range dependence," Annals of Statistics , 23, 1048-1072.

Robinson, P. M. and J. Hualde (2003), "Cointegration in fractional systems with unknown integration orders," Econometrica , 71, 1727-1766.

Said, S. and D. Dickey (1984), "Testing for unit roots in autoregressive moving average models of unknown order," Biometrika, 71, 599-608. 
Sephton, P. (2007), "Critical values for the Augmented Efficient Wald Test for fractional unit roots," Mimeo.

Shimotsu, K. (2006), "Exact local Whittle estimation of fractional integration with unknown mean and time trend," QED Working Paper 1061.

Silverberg, G. and B. Verspagen (2001), "A note on Michelacci and Zaffaroni, long memory and time series of economic growth," University of Maastricht, Mimeo.

Sowell, F.B.(1992), "Maximum likelihood estimation of stationary univariate fractionallyintegrated time-series models," Journal of Econometrics, 53, 165-188.

Tanaka, K. (1999), "The nonstationary fractional unit root," Econometric Theory, 15, 249-264.

Velasco, C. (1999), "Non-stationary log-periodogram regression," Journal of Econometrics, 91, 325-371.

Velasco C. and P. M. Robinson (2000), "Whittle pseudo-maximum likelihood estimation for nonstationary time series," Journal of the American Association, 95 , 1229-1243. 


\section{APPENDIX}

\section{Proof of Theorem 2}

Let us first consider the case where the true value of $d$ is used to compute the test. In this case, under the alternative hypothesis of $\Delta^{d} y_{t}=\varepsilon_{t}$ with $\varepsilon_{t} \sim$ i.i.d. $\left(0, \sigma^{2}\right)$, the $t_{\varphi}(d)$ statistic associated to the coefficient of $z_{t-1}(d)$, in the regression of $\Delta y_{t}$ on $z_{t-1}(d)$ can be written as,

$$
T^{-1 / 2} t_{\varphi}(d)=\frac{\sum_{t=2}^{T} \Delta y_{t} z_{t-1}(d) / T}{\left(\left(\sum_{t=2}^{T}\left(\Delta y_{t}-\widehat{\varphi} z_{t-1}(d)\right)^{2} / T\right)\left(\sum_{t=2}^{T} z_{t-1}^{2}(d) / T\right)\right)^{1 / 2}} .
$$

Using the results collected in Baillie (1996) stating that, if $\Delta^{b} y_{t}=\varepsilon_{t}$ with $b>-1$, then the variance $\left(\gamma_{0}\right)$ and the autocorrelation of order $j\left(\rho_{j}\right)$ of $y_{t}$ satisfy $\gamma_{0}=\sigma^{2} \Gamma(1-2 b) / \Gamma^{2}(1-b)$ and $\rho_{j}=[\Gamma(j+b)(1-b) /(\Gamma(j-b+1) \Gamma(b))]$. In the previous case, where $\Delta y_{t} \sim I(d-1)$ (hence $b=d-1$ ), it is easy to check that the numerator of $T^{-1 / 2} t_{\varphi}(d)$ converges in probability to

$$
\frac{\sum_{t=2}^{T} \Delta y_{t} z_{t-1}(d)}{T}=\frac{\sum_{t=2}^{T}\left(\Delta^{1-d} \varepsilon_{t}\right)\left(\varepsilon_{t}-\Delta^{1-d} \varepsilon_{t}\right)}{(1-d) T} \stackrel{p}{\rightarrow} \frac{\sigma^{2}}{1-d}\left[1-\frac{\Gamma(3-2 d)}{\Gamma^{2}(2-d)}\right],
$$

whereas the two terms in the denominator converge to

$$
\frac{\sum_{t=2}^{T} z_{t-1}^{2}(d)}{T}=\frac{\sum_{t=2}^{T}\left(\varepsilon_{t}-\Delta^{1-d} \varepsilon_{t}\right)^{2}}{(1-d)^{2} T} \stackrel{p}{\rightarrow} \frac{\sigma^{2}}{(1-d)^{2}}\left[\frac{\Gamma(3-2 d)}{\Gamma^{2}(2-d)}-1\right]
$$

and

$$
\frac{\sum_{t=2}^{T}\left(\Delta y_{t}-\widehat{\varphi} z_{t-1}(d)\right)^{2}}{T} \stackrel{p}{\rightarrow} \sigma^{2}
$$

Replacing the previous limits in the expression for $T^{-1 / 2} t_{\varphi}(d)$ yields

$$
T^{-1 / 2} t_{\varphi}(d) \stackrel{p}{\rightarrow}-\left(\frac{\Gamma(3-2 d)}{\Gamma^{2}(2-d)}-1\right)^{1 / 2} \equiv c_{E F D F}(d)
$$


Next, we examine the case where a $T^{\kappa}-$ consistent estimator of $d, \hat{d}_{T}$, for some $d>0.5$ with $\kappa>0$, is employed to construct the test. In this case, provided

$$
T^{-1 / 2} t_{\phi}(d)-T^{-1 / 2} t_{\phi}\left(\hat{d}_{T}\right)=o_{p}(1)
$$

the limit of $T^{-1 / 2} t_{\phi}\left(\hat{d}_{T}\right)$ would also be given by expression $(A 1)$. Following $L V$, we consider the most critical component in this expression, i.e., the numerator of the difference in $(A 2)$, given by

$$
T^{-1}\left(\sum_{t=1}^{T} \Delta y_{t} z_{t-1}(d)-\sum_{t=1}^{T} \Delta y_{t} z_{t-1}\left(\hat{d}_{T}\right)\right) .
$$

Proceeding as Robinson and Hualde (2003), we just need to show that expression

$$
T^{-1}\left(\sum_{t=2}^{T}\left(\Delta^{1-d} \varepsilon_{t}\right) \varepsilon_{t}-\sum_{t=2}^{T}\left(\Delta^{1-\widehat{d}_{T}} \varepsilon_{t}\right) \varepsilon_{t}\right)
$$

tends to zero in probability. It is straightforward to see that

$\frac{\sum_{t=1}^{T}\left(\Delta^{1-d} \varepsilon_{t}\right) \varepsilon_{t}}{T}=\frac{\sum_{t=1}^{T}\left(\varepsilon_{t}+\pi_{1}(1-d) \varepsilon_{t-1}+\pi_{2}(1-d) \varepsilon_{t-2}+\ldots+\pi_{t-1}(1-d) \varepsilon_{1}\right) \varepsilon_{t}}{T} \stackrel{p}{\rightarrow} \sigma^{2}$

since all cross-products tend to zero in probability. As for the second term in $(A 3)$, it can be written as

$$
\frac{\sum \varepsilon_{t}^{2}}{T}+T^{-1} \sum_{t=1}^{T}\left(\sum_{i=1}^{t-1} \sum_{j=1}^{t-1} \pi_{i}(1-d) \pi_{j}\left(\hat{d}_{T}-d\right) \varepsilon_{t-i} \varepsilon_{t-j}\right)
$$

where the first term tends to $\sigma^{2}$. By applying similar steps to those considered in LV (2007, expressions (26)-(28) in appendix 1), it is easy to show that the second term tends to zero in probability. Hence, it follows that $(A 3)$ tends to zero in probability and the desired result follows.

Likewise, the FDF test is based on the t-ratio

$$
T^{-1 / 2} t_{\phi}\left(\tilde{d}_{T}\right)=\frac{\sum \Delta y_{t} \Delta^{\tilde{d}_{T}} y_{t-1} / T}{\left(\left(\sum\left(\Delta y_{t}-\widehat{\phi} \Delta^{\tilde{d}_{T}} y_{t-1}\right)^{2} / T\right)\left(\sum\left(\Delta^{\tilde{d}_{T}} y_{t-1}\right)^{2} / T\right)^{1 / 2}\right.}
$$


As before, when the true value of $d$ is used as input then, by the Law of Large Numbers (LLN), the numerator tends to $(d-1) \sigma^{2}$. With respect to the denominator, we have that $T^{-1} \sum\left(\Delta y_{t}\right)^{2} \stackrel{p}{\rightarrow} \sigma^{2} \Gamma(3-2 d) /(\Gamma(2-d))^{2}$ and $\hat{\phi} \stackrel{p}{\rightarrow}(d-1)$. Combining these results, yields

$$
T^{-1 / 2} t_{\hat{\phi}}(d) \stackrel{p}{\rightarrow} \frac{(d-1) \Gamma(2-d)}{\left[\Gamma(3-2 d)-(d-1)^{2} \Gamma^{2}(2-d)\right]^{1 / 2}} \equiv c_{F D F}(d) .
$$

If a consistent estimate of $d, \hat{d}_{T}$ is employed to run the test, a similar strategy to that followed above can be used to show that $t_{\hat{\phi}}(d)$ also converges to $(A 5)$.

Finally, by the LLN, the LM test defined in (4), multiplied by $T^{-1 / 2}$, satisfies that,

$$
T^{-1 / 2} L M_{T} \stackrel{p}{\rightarrow} \sqrt{\frac{6}{\pi^{2}}} \sum_{k=1}^{T-1} \frac{1}{k} \rho_{k},
$$

where $\rho_{k}$ is the (population) correlation function of a pure $I(d-1)$ process. Using the formula for the autocorrelations given above, yields

$$
T^{-1 / 2} L M_{T} \stackrel{p}{\rightarrow} \sqrt{\frac{6}{\pi^{2}}} \frac{\Gamma(2-d)}{\Gamma(d-1)} \sum_{j=1}^{\infty} \frac{\Gamma(j+d-1)}{j \Gamma(j-d+2)} \equiv c_{L M}(d) .
$$

\section{Proof of Theorem 3}

We consider first the case where $d \in(0.5,1)$ is a fixed number and then extend the proof to case where it is stochastic. In the general case where $\mu(t)$ is different from zero, the t-statistic on the coefficient $\varphi$ from the OLS regression of $\Delta y_{t}$ on $z_{t-1}$ is a function of $\mu(t)$ given by,

$$
t_{\varphi}(d, \mu(t))=\frac{\sum_{t=2}^{T} \Delta y_{t} z_{t-1}(d)}{\hat{S}_{T}(d) \sqrt{\sum_{t=2}^{T}\left(z_{t-1}(d)\right)}},
$$

where $\hat{S}_{T}^{2}(d)=T^{-1} \sum_{t=2}^{T}\left(\Delta y_{t}-\widehat{\varphi} z_{t-1}(d)\right)^{2}$. We now show that the asymptotic distribution of $(A 6)$ for the case where $\mu(t)$ satisfies Condition $\mathrm{A}$ is the same as in the case where $\mu(t) \equiv 0$. Following the same strategy as LV (2007), we now prove that, for $d \neq 1$,

$$
t_{\varphi}(d, \mu(t))-t_{\varphi}(d, \mu(t) \equiv 0)=o_{p}(1)
$$

which implies that the test computed ignoring the fact that the DGP contains slowly evolving trends has the same asymptotic properties as in the case where $\mu(t) \equiv 0$. 
As in LV, we just analyze the most critical component of $t_{\varphi}(d, \mu(t))$, which is the numerator, since the analysis of the denominator is similar but simpler. Under $H_{0}$, the numerator of $(A 6)$, multiplied by $T^{-1 / 2}(1-d)^{-1}$, is given by,

$$
\begin{aligned}
T^{-1 / 2}(1-d)^{-1} \sum_{t=2}^{T} \Delta y_{t} z_{t-1}(d)=T^{-1 / 2} \sum_{t=2}^{T}\left(\Delta \mu(t)+\varepsilon_{t}\right)\left(\left(\Delta^{d}-\Delta\right) \mu(t)+\left(\Delta^{d-1}-1\right) \varepsilon_{t}\right) \\
=T^{-1 / 2}\left(\sum_{t=2}^{T} \varepsilon_{t}\left(\Delta^{d-1}-1\right) \varepsilon_{t}+\sum_{t=2}^{T}\left(\Delta \mu(t)\left(\Delta^{d}-\Delta\right) \mu(t)\right)+\right. \\
\left.\sum_{t=2}^{T} \Delta \mu(t)\left(\Delta^{d-1}-1\right) \varepsilon_{t}+\sum_{t=2}^{T} \varepsilon_{t}\left(\Delta^{d}-\Delta\right) \mu(t)\right)
\end{aligned}
$$

We now show that if $\mu(t)=t^{\delta}, \delta \in[0,0.5)$ all the terms in (A7) and (A8) but the first, $\left(T^{-1 / 2} \sum_{t=2}^{T} \varepsilon_{t}\left(\Delta^{d-1}-1\right) \varepsilon_{t}\right)$, converge to zero. Any other specification of $\mu(t)$ satisfying Condition A can be dealt with analogously.

To prove this, notice that the terms $t^{\delta}$ and $\Delta^{-\delta} 1_{(t>0)}$ are of the same order of magnitude. This is because $\Delta^{-\delta} 1_{(t>0)}=\sum_{i=0}^{t-1} \pi_{i}(-\delta) \approx c \sum_{i=0}^{t-1} i^{\delta-1}=O\left(t^{\delta}\right)$ (see Davidson, 1994, Theorem 2-27), where $c$ is a constant and the coefficients $\pi_{i}(-\delta)$ are defined at the end of the Introduction.

The second term in (A7) verifies that,

$$
\begin{aligned}
T^{-1 / 2}\left(\sum_{t=2}^{T} \Delta \mu(t) \Delta^{d} \mu(t)-\sum_{t=2}^{T}(\Delta \mu(t))^{2}\right) & \approx T^{-1 / 2}\left(\sum_{t=2}^{T} t^{2 \delta-d-1}-\sum_{t=2}^{T} t^{2(\delta-1)}\right) \\
& =T^{-1 / 2}\left(O\left(T^{2 \delta-d}\right)-O(1)\right) \rightarrow 0,(A
\end{aligned}
$$

if $d>0.5$ and $\delta<0.5$.

With respect to the first term in (A8),

$$
T^{-1 / 2} E\left(\sum_{t=2}^{T} \Delta t^{\delta}\left(\Delta^{d-1}-1\right) \varepsilon_{t}\right)=0
$$

and

$$
T^{-1} \operatorname{Var}\left(\sum_{t=2}^{T} \Delta t^{\delta}\left(\Delta^{d-1}-1\right) \varepsilon_{t}\right) \approx T^{-1}\left(\sigma_{\varepsilon}^{2}+\sigma_{\Delta^{d-1} \varepsilon}^{2}\right) \sum_{t=2}^{T} t^{2(\delta-1)} \rightarrow 0
$$


where $\sigma_{\Delta^{d-1} \varepsilon}^{2}$ denotes the variance of the stationary fractionally integrated process $\Delta^{d-1} \varepsilon_{t}$. Expressions (A10) and (A11) imply that $\sum_{t=2}^{T} \Delta t^{\delta}\left(\Delta^{d-1}-1\right) \varepsilon_{t} \stackrel{p}{\rightarrow} 0$. The same type of argument can be used to show that the second term in (A8) also converges to zero. Therefore, for $d \neq 1$, it follows that

$$
(1-d)^{-1} T^{-1 / 2} \sum_{t=2}^{T} \Delta y_{t} z_{t-1}(d)=(1-d)^{-1} T^{-1 / 2} \sum_{t=2}^{T} \varepsilon_{t}\left(\Delta^{d-1}-1\right) \varepsilon_{t}+o_{p}(1),
$$

which in turn implies that the distribution for the case where the DGP contains slowly evolving trends is the same as that obtained with $\mu(t)=0$ for the case where $d$ is a fixed number $\in(0.5,1)$. Considering an stochastic input for $\widehat{d}_{T}$ amounts to show that

$$
t_{\varphi}(d, \mu(t))-t_{\varphi_{\text {ols }}}\left(\hat{d}_{T}, \mu(t)\right)=o_{p}(1)
$$

where $\hat{d}_{T}$ satisfies the conditions stated in Theorem 1. It is easy to show, following the same strategy as above, that the last three terms computed with the estimated input $\hat{d}_{T}$ converge to zero. Hence, the numerator of $t_{\varphi}(d, \mu(t))-t_{\varphi}\left(\hat{d}_{T}, \mu(t)\right)$ can be written as

$$
(d-1)^{-1} T^{-1 / 2}\left(\sum_{t=2}^{T} \varepsilon_{t}\left(\Delta^{d-1}-1\right) \varepsilon_{t}-\sum_{t=2}^{T} \varepsilon_{t}\left(\Delta^{\widehat{d}_{T}}-1\right) \varepsilon_{t}\right)+o_{p}(1),
$$

and LV (2007, Appendix 1) have shown that the first term of this expression also tends to zero.

The case where $d=1-\gamma / \sqrt{T}$ can be solved in an analogous fashion, taking into account the derivations reported in Appendix 1 of LV (2007). Finally, using the results in DGM and LV, it is straightforward to prove the consistency of the test under fixed alternatives.

\section{Proof of Theorem 4}

We start, as before, by analyzing the case where the input of $z_{t-1}(d), d^{*}$, is fixed. We now show that under $H_{0}: d=1, t_{\varphi}(d, \mu(t)=0)-t_{\varphi}(d, \hat{\mu}(t)) \stackrel{p}{\rightarrow} 0$, where in this case $t_{\varphi}(d, \hat{\mu}(t))$ is given by,

$$
t_{\varphi}(d, \hat{\mu}(t))=\frac{\sum_{t=2}^{T} \widetilde{\Delta y_{t}} \widetilde{z_{t-1}}(d)}{\hat{S}_{T}(d) \sqrt{\sum_{t=2}^{T}\left(\widetilde{z_{t-1}}(d)\right)}},
$$

where $\widetilde{\Delta y_{t}}=\left(\Delta y_{t}-\Delta \hat{\mu}(t)\right), \widetilde{z_{t-1}}(d)=(1-d)^{-1}\left(\Delta^{d-1}-1\right)\left(\Delta y_{t}-\Delta \hat{\mu}(t)\right)$ and $\hat{S}_{T}^{2}(d)=$ $T^{-1} \sum_{t=2}^{T}\left(\widetilde{\Delta y_{t}}-\hat{\varphi} \widetilde{z_{t-1}}(d)\right)^{2}$ and $\mu(t)$ satisfies condition B. 
For simplicity, we consider the DGP with a linear trend

$$
y_{t}=\alpha+\beta t+\Delta^{-d} \varepsilon_{t}, d \leq 1
$$

since any other power of $\mathrm{t}^{\delta}$ for $\delta \geq 0.5$ can be handled similarly. Let $\hat{\beta}$ be the OLS estimate of $\beta$, computed after taking first differences in (A8). Then, $\hat{\beta}=\overline{\Delta y_{t}}$, where $\overline{\Delta y_{t}}$ is the sample mean of $\Delta y_{t}$. Notice that under $(A 13), \hat{\beta}$ is a $T^{3 / 2-d}$-consistent estimator of $\beta$ (see Hosking, 1996). As in Theorem 2, we analyze the numerator of $t_{\varphi}$ since the analysis of the denominator is similar but simpler.

The numerator of $t_{\varphi}(d, \hat{\mu}(t))$ multiplied by $(1-d)$ is given by,

$$
\left.T^{-1 / 2}(1-d) \sum_{t=2}^{T} \widetilde{\Delta y_{t}} \widetilde{z_{t-1}}=T^{-1 / 2} \sum_{t=2}^{T} \varepsilon_{t}\left(\Delta^{d-1}-1\right) \varepsilon_{t}\right)+T^{-1 / 2} A_{t}
$$

where

$\left.T^{-1 / 2} A_{t}=T^{-1 / 2}(\beta-\hat{\beta})\left(\sum\left(\Delta^{d-1}-1\right) \varepsilon_{t}\right)+(\beta-\hat{\beta}) \sum_{t=2}^{T} \tau_{t}(d)+\left(\sum_{t=2}^{T}\left(\tau_{t}(d)-1\right) \varepsilon_{t}\right)\right)$,

with $\tau_{t}(\varrho)=\sum_{i=0}^{t-1} \pi_{i}(\varrho)$ and the coefficients $\pi_{i}(\varrho)$ are defined at the end of the Introduction. It is easy to check that, under $H_{0}$,

$$
T^{-1 / 2} A_{t}\left(d_{1}\right)=O_{p}\left(T^{-1}\right)\left(o_{p}(T)+O_{p}\left(T^{-1 / 2}\right) O\left(T^{1-d}\right)+O_{p}\left(T^{1 / 2}\right)\right) \stackrel{p}{\rightarrow} 0 .
$$

The same strategy can be used to show that the denominator of $t_{\varphi}(d, \hat{\mu}(t))$ equals the denominator of $t_{\varphi}(d, \mu(t)=0)$ plus some terms that go to zero in probability. This implies that $t_{\varphi}(d, \hat{\mu}(t)) \stackrel{w}{\rightarrow} N(0,1)$. When $d$ is replaced by $\widehat{d}_{T}$, if $t_{\varphi}(d, \hat{\mu}(t))-t_{\varphi}\left(\hat{d}_{T}, \hat{\mu}(t)\right)=$ $o_{p}(1)$, then the asymptotic distribution corresponding to $t_{\varphi}\left(\hat{d}_{T}, \mu(t)\right)$ would be the same as that of $t_{\varphi}(d, \mu(t))$. Following the same steps as above, it is straightforward to show that $T^{-1 / 2} A_{t}\left(\hat{d}_{T}\right)$ tends to zero. Then, the numerator of $(1-d)\left(t_{\varphi}(d, \mu(t))-t_{\varphi}\left(\hat{d}_{T}, \mu(t)\right)\right)$ can be written as,

$$
(d-1)^{-1} T^{-1 / 2}\left(\sum_{t=2}^{T} \varepsilon_{t}\left(\Delta^{d-1}-1\right) \varepsilon_{t}-\sum_{t=2}^{T} \varepsilon_{t}\left(\Delta^{\widehat{d}_{T}-1}-1\right) \varepsilon_{t}\right)+o_{p}(1)
$$

and LV (2007) have shown that this expression tends to zero under the conditions stated in Theorem 1. Similar results can be easily obtained for the denominator. Hence, $t_{\varphi}\left(\hat{d}_{T}, \hat{\mu}(t)\right) \stackrel{w}{\rightarrow}$ $N(0,1)$. 
Again, the case where $d=1-\gamma / \sqrt{T}$ can be solved in a similar manner, taking into account the derivations reported in Appendix 1 of LV(2007). Likewise, using the results in DGM and $\mathrm{LV}$, the proof of the consistency of the test under fixed alternatives is straightforward.

\section{Proof of Theorem 5}

The proof of this theorem can be easily constructed along the lines of Appendix 2 in LV (2007) and Theorems 2 and 3 above. Therefore, it is omitted. 


\section{TABLES}

TABLE 1

Size And Power(*) of EFDF, LM And ELW tests

DGP: $y_{t}=\alpha+\beta t+\Delta^{-d} \varepsilon_{t} ; 5 \%$ S.L.

$d=1-\gamma / \sqrt{T} ; \alpha=1 ; \varepsilon_{t} \sim N(0,1)$

\begin{tabular}{cccccccccccccc}
\hline \hline & \multirow{2}{*}{$\operatorname{EFDF}_{\mu,(\beta=0)}$} & \multicolumn{2}{c}{$\operatorname{EFDF}_{\tau,(\beta=0.2)}$} & \multicolumn{2}{c}{$\mathrm{LM}_{\mu,(\beta=0)}$} & $\mathrm{LM}_{\tau,(\beta=0.2)}$ & \multicolumn{2}{c}{$\mathrm{ELW}_{\mu},(\beta=0)$} & \multicolumn{3}{c}{$\mathrm{ELW}_{\tau,(\beta=0.2)}$} \\
\hline$\gamma \backslash T$ & 100 & 400 & 100 & 400 & 100 & 400 & 100 & 400 & 100 & 400 & 100 & 400 \\
\hline 0 & 0.061 & 0.045 & 0.074 & 0.087 & 0.031 & 0.029 & 0.049 & 0.046 & 0.071 & 0.075 & 0.072 & 0.074 \\
0.5 & 0.116 & 0.195 & 0.123 & 0.164 & 0.111 & 0.190 & 0.100 & 0.162 & 0.091 & 0.090 & 0.086 & 0.096 \\
1 & 0.287 & 0.378 & 0.252 & 0.328 & 0.273 & 0.369 & 0.237 & 0.324 & 0.150 & 0.158 & 0.125 & 0.154 \\
2 & 0.728 & 0.834 & 0.649 & 0.774 & 0.681 & 0.803 & 0.612 & 0.748 & 0.361 & 0.353 & 0.301 & 0.339 \\
5 & 1.000 & 1.000 & 1.000 & 1.000 & 0.980 & 0.991 & 0.951 & 0.962 & 0.953 & 0.932 & 0.909 & 0.940 \\
\hline
\end{tabular}

$\left(^{*}\right)$ Size-adjusted power. Number of replications: 10000.

\section{TABLE 2}

POWER(*) OF EFDF, LM AND ELW TESTS

DGP: $y_{t}=\alpha+\beta t+\Delta^{-d} \varepsilon_{t}, 5 \%$ S.L.

\begin{tabular}{cccccccc}
\multicolumn{3}{c}{$d=1-\gamma / \sqrt{T} ; \alpha=1 ; \beta=0.2 ; \varepsilon_{t} \sim \chi_{1}^{2}$} \\
\hline \multicolumn{1}{c}{$\mathrm{EFDF}_{\tau}$} & \multicolumn{2}{c}{$\mathrm{LM}_{\tau}$} & \multicolumn{2}{c}{$\mathrm{ELW}_{\tau}$} \\
\hline$\gamma / T$ & 100 & 400 & 100 & 400 & 100 & 400 \\
\hline 0.5 & 0.151 & 0.150 & 0.127 & 0.143 & 0.088 & 0.093 \\
1 & 0.321 & 0.354 & 0.272 & 0.331 & 0.166 & 0.172 \\
2 & 0.737 & 0.806 & 0.704 & 0.765 & 0.376 & 0.387 \\
\hline
\end{tabular}

$\left.{ }^{*}\right)$ Size-adjusted power; Number of replications: 10000. 
TABLE 3

Size And Power ${ }^{(*)}$ of AEFDF, LM And ELW Tests

DGP: $y_{t}=\alpha+\beta t+\Delta^{-d} \epsilon_{t} /\left(1-\phi_{1} L\right) ; 5 \%$ S.L.

$d=1-\gamma / \sqrt{T} ; \phi_{1}=0.2 ; \alpha=1 ; \beta=0.2 ; \phi_{1}=0.2 ; \varepsilon_{t} \sim N(0,1)$

\begin{tabular}{cccccccccccccc}
\hline \hline & $\mathrm{AEFDF}_{\mu,(\beta=0)}$ & \multicolumn{2}{c}{$\mathrm{AEFDF}_{\tau,(\beta=0.2)}$} & \multicolumn{2}{c}{$\mathrm{LM}_{\mu,(\beta=0)}$} & $\mathrm{LM}_{\tau,(\beta=0.2)}$ & $\mathrm{ELW}_{\mu,(\beta=0)}$ & $\mathrm{ELW}_{\tau,(\beta=0.2)}$ \\
\hline$\gamma \backslash \mathrm{T}$ & 100 & 400 & 100 & 400 & 100 & 400 & 100 & 400 & 100 & 400 & 100 & 400 \\
\hline 0 & 0.061 & 0.066 & 0.075 & 0.065 & 0.033 & 0.031 & 0.029 & 0.050 & 0.058 & 0.056 & 0.057 & 0.053 \\
0.5 & 0.093 & 0.091 & 0.075 & 0.085 & 0.079 & 0.077 & 0.070 & 0.079 & 0.097 & 0.072 & 0.083 & 0.070 \\
1 & 0.139 & 0.154 & 0.108 & 0.155 & 0.112 & 0.151 & 0.091 & 0.134 & 0.139 & 0.121 & 0.121 & 0.118 \\
2 & 0.328 & 0.352 & 0.237 & 0.342 & 0.285 & 0.338 & 0.224 & 0.314 & 0.321 & 0.278 & 0.312 & 0.291 \\
5 & 0.952 & 0.973 & 0.840 & 0.942 & 0.891 & 0.947 & 0.811 & 0.914 & 0.910 & 0.898 & 0.814 & 0.890 \\
\hline
\end{tabular}

$\left(^{*}\right)$ Size-adjusted power; Number of replications: 10000. 


\section{TABLE 4a}

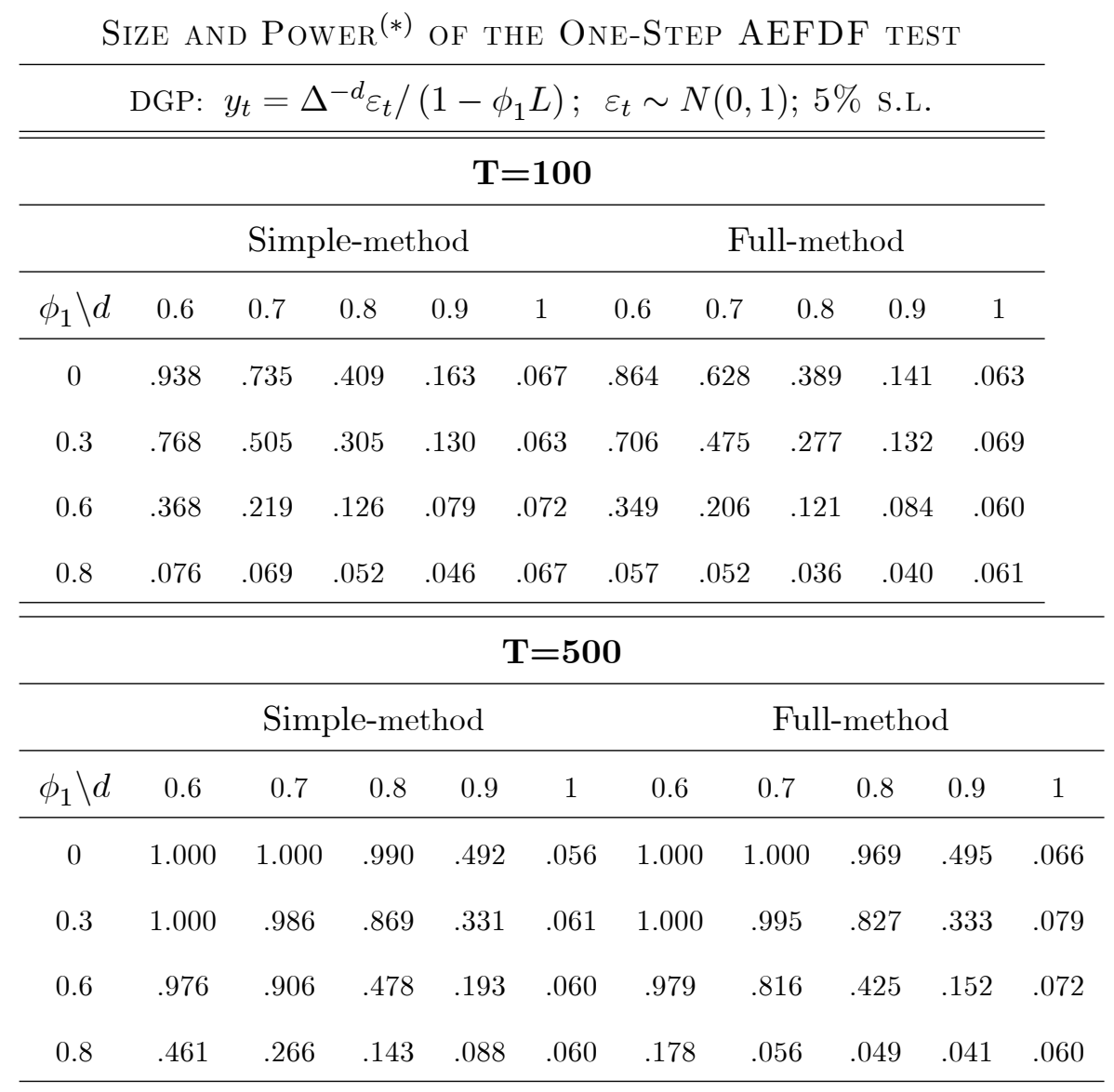

$\left(^{*}\right)$ Size-adjusted power. Number of replications: 10000.

Note.- Simple-method consists of regressing $y_{t}$ on $z_{t-1}(d)$ and lags of $\Delta y_{t}$. For this table, one and two lags of $\Delta y_{t}$ have been included for $\mathrm{T}=100$ and $\mathrm{T}=500$, respectively. Full-method, in general, is based on regression (21). For the particular DGP of this table is based on regression (22). 
TABLE 4b

Size And Power(*) of the Two-Step AEFDF test

\begin{tabular}{cccccccccccc}
\hline \multicolumn{1}{c}{ DGP: } & $y_{t}=\Delta^{-d} \varepsilon_{t} /\left(1-\phi_{1} L\right) ;$ & $\varepsilon_{t} \sim N(0,1) ; 5 \%$ S.L. \\
\hline \hline \multicolumn{1}{c}{$\mathbf{T}=\mathbf{1 0 0}$} \\
\hline$\phi_{1} \backslash d$ & 0.6 & 0.7 & 0.8 & 0.9 & 1 & 0.6 & 0.7 & 0.8 & 0.9 & 1 \\
\hline 0 & .931 & .712 & .398 & .157 & .077 & 1.000 & .999 & .980 & .516 & .065 \\
0.3 & .762 & .503 & .268 & .119 & .073 & 1.000 & .999 & .868 & .344 & .063 \\
0.6 & .372 & .218 & .126 & .078 & .068 & .996 & .919 & .437 & .158 & .062 \\
0.8 & .052 & .048 & .040 & .038 & .065 & .363 & .152 & .062 & .045 & .061 \\
\hline
\end{tabular}

$\left(^{*}\right)$ Size-adjusted power. Number of replications: 10000.

TABLE 5

\begin{tabular}{ccc} 
Estimates & OF $\widehat{\beta}$ AND ROBUST & S.E $(\widehat{\beta})$ IN $\Delta y_{t}=\beta+u_{t}$ \\
\hline \hline Country & Mean & Robust s.e. \\
\hline Australia & 0.0148 & 0.004 \\
Belgium & 0.015 & 0.005 \\
Canada & 0.0195 & 0.005 \\
Denmark & 0.0184 & 0.008 \\
France & 0.0185 & 0.006 \\
Germany & 0.0176 & 0.007 \\
Italy & 0.0192 & 0.006 \\
Netherlands & 0.0154 & 0.006 \\
Norway & 0.0221 & 0.006 \\
UK & 0.0143 & 0.003 \\
USA & 0.0186 & 0.005 \\
Spain & 0.0199 & 0.005 \\
Sweden & 0.0193 & 0.005 \\
\hline
\end{tabular}


TABLE 6

AEFDF TEST

\begin{tabular}{ccc}
\multicolumn{3}{c}{$H_{0}: I(1)$ vs. $H_{A}: d<1$} \\
\hline \hline Country & $t_{\varphi}\left(\widehat{d}_{T}\right)$ & $\widehat{d}_{T}($ s.e. $=0.10)$ \\
\hline Australia & -1.02 & 1.10 \\
Belgium & -0.74 & 0.98 \\
Canada & $-2.58^{*}$ & 0.80 \\
Denmark & -0.72 & 0.99 \\
France & $-1.82^{*}$ & 1.08 \\
Germany & $-1.94^{*}$ & 0.83 \\
Italy & -0.18 & 0.98 \\
Netherlands & $-1.76^{*}$ & 0.92 \\
Norway & -1.03 & 0.98 \\
UK & $-1.94^{*}$ & 0.87 \\
USA & $-3.50^{*}$ & 0.63 \\
Spain & -0.17 & 1.18 \\
Sweden & -0.07 & 1.12
\end{tabular}

$\left(^{*}\right)$ denotes rejection at the $5 \%$ s.l. 